\title{
Pituitary Adenylate Cyclase-Activating Polypeptide Attenuates Brain Edema by Protecting Blood-Brain Barrier and Glymphatic System After Subarachnoid Hemorrhage in Rats
}

\author{
Yuanjian Fang ${ }^{1} \cdot$ Hui Shi $^{2} \cdot$ Reng Ren ${ }^{1} \cdot$ Lei Huang ${ }^{3,4} \cdot$ Takeshi Okada $^{3,4} \cdot$ Cameron Lenahan $^{3,5} \cdot$ Marcin Gamdzyk $^{3}$. \\ Zachary D. Travis ${ }^{3} \cdot$ Qin Lu $^{6} \cdot$ Lihui Tang $^{1} \cdot$ Yi Huang ${ }^{1} \cdot$ Keren Zhou ${ }^{1} \cdot$ Jiping Tang ${ }^{3,4,7} \cdot$ Jianmin Zhang ${ }^{1}$. \\ John H. Zhang ${ }^{3,4,7}$
}

Accepted: 28 August 2020 / Published online: 11 September 2020

(C) The American Society for Experimental NeuroTherapeutics, Inc. 2020

\begin{abstract}
Brain edema is a vital contributor to early brain injury after subarachnoid hemorrhage (SAH), which is responsible for prolonged hospitalization and poor outcomes. Pharmacological therapeutic targets on edema formation have been the focus of research for decades. Pituitary adenylate cyclase-activating polypeptide (PACAP) has been shown to participate in neural development and brain injury. Here, we used PACAP knockout CRISPR to demonstrate that endogenous PACAP plays an endogenous neuroprotective role against brain edema formation after SAH in rats. The exogenous PACAP treatment provided both short- and longterm neurological benefits by preserving the function of the blood-brain barrier and glymphatic system after SAH. Pretreatment of inhibitors of PACAP receptors showed that the PACAP-involved anti-edema effect and neuroprotection after SAH was facilitated by the selective PACAP receptor (PAC1). Further administration of adenylyl cyclase (AC) inhibitor and sulfonylurea receptor 1 (SUR1) CRISPR activator suggested that the AC-cyclic adenosine monophosphate (cAMP)-protein kinase A (PKA) axis participated in PACAP signaling after SAH, which inhibited the expression of edema-related proteins, SUR1 and aquaporin4 (AQP4), through SUR1 phosphorylation. Thus, PACAP may serve as a potential clinical treatment to alleviate brain edema in patients with SAH.
\end{abstract}

Key Words Subarachnoid hemorrhage $\cdot$ brain edema $\cdot$ pituitary adenylate cyclase-activating polypeptide $\cdot$ blood-brain barrier . glymphatic system

Yuanjian Fang and Hui Shi contributed equally to this work.

Electronic supplementary material The online version of this article (https://doi.org/10.1007/s13311-020-00925-3) contains supplementary material, which is available to authorized users.

Jianmin Zhang

zjm135@zju.edu.cn

$\square$ John H. Zhang

johnzhang3910@yahoo.com

1 Department of Neurosurgery, The Second Affiliated Hospital, School of Medicine, Zhejiang University, 88 Jiefang Road, Zhejiang 310009, Hangzhou, China

2 Department of Neurosurgery, Yongchuan Hospital, Chongqing Medical University, Chongqing, China

3 Department of Neurosurgery, Loma Linda University, Loma Linda, California, USA
4 Department of Physiology and Pharmacology, Loma Linda University, 11041 Campus St, Risley Hall, Room 219, Loma Linda, California 92354, USA

5 Burrell College of Osteopathic Medicine, Las Cruces, New Mexico, USA

6 Department of Neurosurgery, Sir Run Run Shaw Hospital, School of Medicine, Zhejiang University, Hangzhou, Zhejiang, China

Department of Anesthesiology, Loma Linda University, Loma Linda, California, USA 


\section{Introduction}

Brain edema is an important contributor to early brain injury after subarachnoid hemorrhage (SAH), which is responsible for both late neurologic function decline and poor outcome in SAH patients $[1,2]$. The severity of cerebral edema directly predicted the prognosis of SAH patients [2]. Although treatments, such as osmotherapy and decompressive craniectomy (for sever edema), are applied in the current clinical setting to alleviate cerebral edema, the effect remains limited to improvement of outcomes. Developing pharmacological therapeutics targeting edema formation at the cellular and molecular level is encouraged for the treatment of SAH patients [3].

The cerebral edema formation after stroke is currently characterized as cytotoxic, ionic, or vasogenic edema $[4,5]$. Cytotoxic edema is initiated within minutes after hemorrhage and is mainly caused by the immediately influx of osmolites (mainly ions) and water from the interstitial fluid (ISF) into the astrocytes [6]. A recent hypothesis pertaining to the glymphatic system suggested that ISF, mainly derived from cerebrospinal fluid (CSF) via Virchow-Robin spaces (also described as perivascular space), functions as a source of ions and water $[7,8]$. Further dislocated astrocytic aquaporin4(AQP4) facilitates the accumulation of water in the brain parenchyma, aggravating brain swelling $[9,10]$. Following brain injury, it was proposed that upregulated cation channel protein sulfonylurea receptor 1 (SUR1) may cause glymphatic dysfunction by recruiting AQP4 to form an ion/water channel complex that leads to a bulk water influx during astrocytic swelling and irregular CSF movement in the parenchyma [11]. Ionic edema occurs immediately following cytotoxic edema. It is driven by the cytotoxic edema-caused ionic gradient between the vascular compartment and ISF [12]. Ions and water cross the brain endothelial cells via endothelial ion channels and transporters, such as SUR1/transient receptor potential-melastatin 4, sodium-potassium-chloride cotransporter 1 , and $\mathrm{Na}+\mathrm{H}+$ exchanger [13]. These channels also participate in endothelial cell dysfunction, which further exacerbates vasogenic edema $[14,15]$. The impaired bloodbrain barrier (BBB) allows extravasation of water and plasma proteins, such as $\operatorname{IgG}$ and albumin, from the capillary lumen into the brain interstitial compartment, exacerbating osmotic imbalance and brain water accumulation [3].

Pituitary adenylate cyclase-activating polypeptide (PACAP) is a neuropeptide belong to the vasoactive intestinal peptide (VIP)/glucagon/secretin family and is widely distributed throughout the central nervous system (CNS), including the hypothalamus, pituitary, hippocampus, and cortex [16, 17]. Encoded by the ADCYAP1 gene, PACAP exists as two different isoforms, PACAP27 and PACAP38, which are named according to the number of amino acid peptides at the N-terminus [17]. Two types of $\mathrm{G}$ protein coupled receptors are shared between PACAP and VIP due to the high homology. The first type is the selective receptor, namely PACAP receptor 1 (PAC1), which exhibits 100-fold affinity for PACAP over VIP. The second type is the nonselective receptor, namely VIP receptor (VPAC) 1 and VPAC2, which have a similar affinity for PACAP and VIP [18]. Although $\mathrm{PAC} 1$ is particularly abundant in brain regions consistent with PACAP distribution, VPAC1/2 are mainly expressed in regions outside of the brain. Thus, PAC1 is considered the primary functional receptor of PACAP in the CNS $[16,18]$.

PACAP has multiple physiological functions in the CNS. It acts not only as a neurotrophic factor, a neurotransmitter, and an immuno-modulator in neural development but also as a neuroprotectant in response to stress [17]. Accumulating evidence shows that PACAP engaged in acute and chronic CNS pathophysiologic conditions, including stroke [19]. In a clinical study of SAH patients, higher plasma PACAP levels were associated with a better prognosis [20]. Additionally, the PACAP has been shown to attenuate various types of edema, including ischemia-induced cerebral edema [21, 22], skin edema [23], and macular edema [24]. The potential mechanism of protection involves the modulation of ion and water channel proteins $[25,26]$. Moreover, the classical pathway of PACAP signaling, adenylyl cyclase (AC)-cyclic adenosine monophosphate (cAMP)-protein kinase A (PKA) [19], has a potent effect on regulating cerebral edema-related protein SUR1 [27, 28], a key protein contributing to the dysfunction of the glymphatic system and BBB.

Herein, we explored the role of endogenous PACAP on cerebral edema formation and investigated the treatment effects of exogenous PACAP on cerebral edema formation and underlying mechanisms of protection pertaining to the function of the glymphatic system and BBB after SAH in rats.

\section{Methods}

\section{Experiment Design}

The study was divided into 5 experiments (Fig. 1).

Experiment 1. Role of Endogenous PACAP on Cerebral Edema Formation After SAH PACAP knockout CRISPR was used to explore the role of PACAP on brain edema and neurological deficits after SAH. Forty rats were randomly assigned to the following four groups ( $n=10$ /group): Sham, SAH, SAH + control CRISPR, and SAH + PACAP knockout CRISPR. Neurological scores were measured $24 \mathrm{~h}$ after SAH for each rat. Intracerebral pressure (ICP, $n=10$ /group) and brain water content ( $n=6$ /group) were measured $24 \mathrm{~h}$ after SAH to evaluate brain edema. Western blot was used to assess protein levels of PACAP, SUR1, and AQP4 in ipsilateral hemisphere brain tissues ( $n=4 /$ group). 

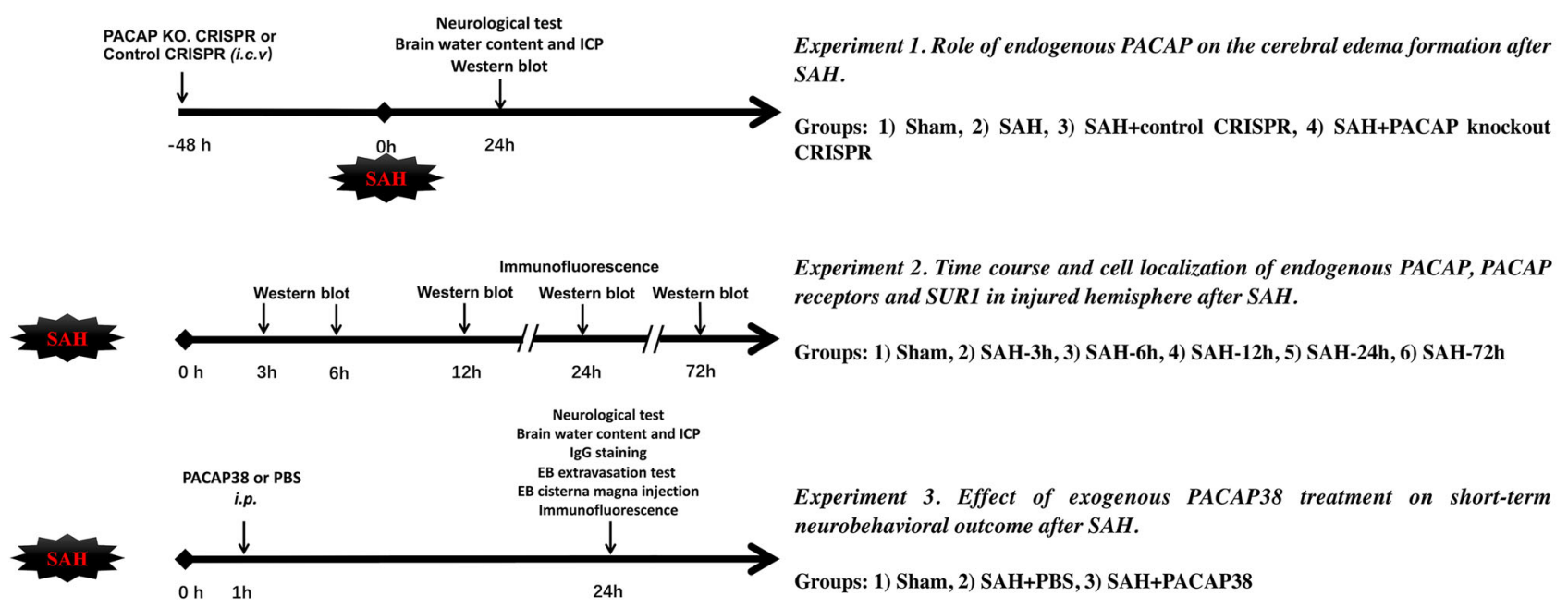

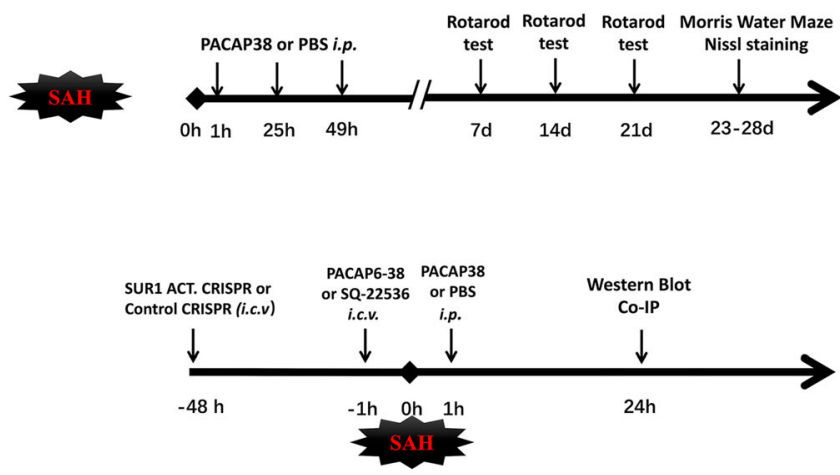

Fig. 1 The study design

Experiment 2. Time Course and Cell Localization of Endogenous PACAP, PACAP Receptors, and SUR1 in the Ipsilateral Hemisphere After SAH Thirty-six rats were randomly assigned to the following 6 groups ( $n=6 /$ group): sham, SAH-3 h, SAH-6 h, SAH-12 h, SAH- 24 h, and SAH-72 h. Western blots were used to evaluate the protein changes of PACAP, PAC1, VPAC1, VPAC2, and SUR1 in ipsilateral hemisphere brain tissues after SAH. Additionally, 8 rats from sham and SAH-24 h ( $n=4$ /group) were used for double immunofluorescence staining to explore cell localization and quantification of PACAP, PAC1, and VPAC1/VPAC2. Because previous study has demonstrated that 1) AQP4 expressions increased $6 \mathrm{~h}$ and maintained high levels at $72 \mathrm{~h}$ after SAH and 2) its cellular expressions were specifically localized on astrocytes after SAH [29], we did not measure AQP4 time course and cellular localization in this part.

Experiment 3. Effect of Exogenous PACAP38 Treatment on Short-Term Neurobehavioral Outcome After SAH Thirty rats were randomly assigned to the following 5 groups $(n=6 /$ group): sham, SAH + PBS, SAH + PACAP38 $(10 \mu \mathrm{g} / \mathrm{kg})$, $\mathrm{SAH}+\mathrm{PACAP} 38(30 \mu \mathrm{g} / \mathrm{kg})$, and $\mathrm{SAH}+\mathrm{PACAP} 38$ $(90 \mu \mathrm{g} / \mathrm{kg})$. Neurological scores, ICP, and brain water content
Experiment 4. Effect of exogenous PACAP38 treatment on long-term neurobehavioral outcome after $S A H$.

Groups: 1) Sham, 2) SAH+PBS, 3) SAH+PACAP38

Experiment 5. Potential neuroprotective molecular mechanism of PACAP after SAH.

PartA-Groups: 1) Sham, 2) SAH+PBS+DMSO, 3) SAH+PACAP38+DMSO, 4) SAH+PACAP38+PACAP6-38, 5) SAH+PACAP38+SQ22536

PartB-Groups: 1) SAH+PBS+Control CRISPR, 2) SAH+PBS+SUR1 Activation CRISPR, 3) SAH+PACAP38+Control CRISPR, 4)SAH+PACAP38+SUR1 Activation CRISPR

were measured $24 \mathrm{~h}$ after SAH. To further validate PAC1 as the functional receptor of PACAP, 4 additional groups $(n=6 /$ group) were included using SAH rats: $\mathrm{PBS}+\mathrm{DMSO}$, PACAP38 + DMSO, PACAP38 + PACAP6-38, and PACAP38 + VIP6-28. Lastly, to study the role of exogenous PACAP38 on BBB and glymphatic functions, another set of 36 rats were randomly divided into the following 3 groups ( $n=12$ /group): sham, SAH + PBS, and SAH + PACAP38. IgG staining and vascular Evans blue (EB) extravasation tests (intraperitoneal injection) were used to evaluate BBB function; CSF EB spread/clearance assessment (cisterna magna injection) and double immunofluorescence staining of perivascular AQP4 were used to evaluate glymphatic function.

Experiment 4. Effect of Exogenous PACAP38 Treatment on Long-Term Neurobehavioral Outcome After SAH Thirty rats were randomly assigned to the following 3 groups $(n=10 /$ group): sham, SAH + PBS, and SAH + PACAP38. The rotarod tests were conducted on days 7, 14, and 21 after SAH. The Morris water maze was conducted from days 23 to 28 after SAH. The Nissl staining was conducted to assess the ipsilateral hippocampal neuron loss and cerebral 
ventricular volumes and cortical thickness for post-SAH hydrocephalus on day 28 after SAH.

\section{Experiment 5. Potential Neuroprotective Molecular} Mechanism of PACAP After SAH This part was divided into 2 subexperiments: part A, explore the role of $\mathrm{PAC} / \mathrm{AC} / \mathrm{cAMP} /$ PKA pathway in PACAP signaling-provided neuroprotection. A total of 30 rats were randomly assigned to the following 5 groups ( $n=6$ /group): sham, $\mathrm{SAH}+\mathrm{PBS}+\mathrm{DMSO}, \mathrm{SAH}+$ PACAP38 + DMSO, SAH + PACAP38 + PACAP6-38 and SAH + PACAP38 + SQ22536. Part B, further explore the role of SUR1 in PACAP-provided neuroprotection. A total of 24 SAH rats were randomly assigned to the following 4 groups ( $n=6$ /group): PBS + control CRISPR, PBS + SUR1 activation CRISPR, PACAP38 + control CRISPR, and PACAP38 + SUR1 activation CRISPR. Western blot was used to evaluate the protein changes of PACAP/PAC1/AC/cAMP/PKA signaling pathway, edema-related proteins (AQP4, SUR1), and BBB-related proteins (MMP-9, ZO-1) within the ipsilateral hemisphere. Co-IP of AQP4-SUR1 and phosph-SUR1 was conducted to evaluate the changes of the AQP4-SUR1 complex and phosphorylated SUR1.

\section{Animals}

All experimental procedures involving animals were approved by the Institutional Animal Care and Use Committee of Loma Linda University and were conducted in compliance with protocols established by the National Institutes of Health Guide for the Care and Use of Laboratory Animals. Adult male Sprague-Dawley rats weighing 280-320 g were used for this experiment. All rats were housed in a facility with a 12-h/12-h light/dark cycle and were given free access to water and standard rodent chow.

\section{SAH Model and SAH Grading}

The endovascular perforation SAH model was conducted as previously described [30]. Briefly, isoflurane-anesthetized rats were intubated with mechanical ventilation. A sharp 4-0 monofilament was inserted from the left external carotid artery to the internal carotid artery and perforated the bifurcation of the anterior and middle cerebral arteries. Rats in the sham group underwent the same procedures without vessel puncture. Measurements of respiration, heart rate, skin pigmentation, and pedal reflex were recorded intraoperatively every $5 \mathrm{~min}$ to confirm the anesthetic status and prevent distress.

The SAH grading was blindly evaluated by two independent investigators according to the previous study [31]. Briefly, the basal cistern was divided into six segments and scored from 0 to 3 based on the subarachnoid blood clot. The final score combined all segments and ranged from 0 to 18 . If there was no blood present, a score of 0 was assigned. Rats with a score under 9 were excluded from this study.

\section{Drug Administration}

Two administration routes were used including intracerebroventricular (i.c.v.) route and intraperitoneal (i.p.) route.

The engineered form of CRISPR-associated (Cas9) protein system (Santa Cruz Biotechnology, Dallas, TX) was used in the study. Briefly, the CRISPR protein Cas9 is directed to genomic target sites by short guide RNAs, where it functions as an endonuclease, and further inactivates or activates gene [32]. PACAP knockout CRISPR (sc-437378, gRNA sequences: CGAAGCCTACCGCAAAGTCT; CTGTGAAGATGCCG TCCGAG; GTAGCACCTACCTGATCCCA), SUR1 activation CRISPR (sc-4237217, gRNA sequence: ACCCAATG CGCACGTGCGCT), and control (scrambled) CRISPR (sc418922) were given ( $2 \mu \mathrm{g} / \mathrm{rat}$, i.c.v.) $48 \mathrm{~h}$ before SAH. Western blot was performed to confirm the effect of knockout and activation CRISPR. Control CRISPR was used to avoided potential bias carried by the off-target effects of CRISPR.

The drugs (Tocris Bioscience, Minneapolis, $\mathrm{MN}$ ), including PACAP6-38 (PAC1 selective inhibitor, $50 \mu \mathrm{g} / \mathrm{kg}$ ), VIP6-28 (VPAC1/VPAC2 nonselective inhibitor, $50 \mu \mathrm{g} / \mathrm{kg}$ ), and SQ22536 (AC selective inhibitor, $2 \mathrm{nmol}$ per rats), were dissolved in $1 \%$ dimethyl sulfoxide (DMSO), and administered via i.c.v. injection $1 \mathrm{~h}$ before surgery.

The i.c.v. administration was conducted as previously described $[3,31]$. Rats were and placed in a stereotaxic frame under isoflurane anesthesia. A precision $10-\mu l$ syringe (Hamilton Company, Reno, NV) was inserted through a burr hole into the left lateral ventricle $(0.9 \mathrm{~mm}$ caudal and $1.5 \mathrm{~mm}$ lateral to the bregma, $3.3 \mathrm{~mm}$ below the horizontal plane of the skull). Drugs or CRISPRs were infused at a rate of $1 \mu \mathrm{l} / \mathrm{min}$ to prevent possible leakage, the needle was removed at 5 min after i.c.v. infusion completion. Lastly, the skull hole was sealed quickly with bone wax, and then the incision site was sutured.

Exogenous PACAP38 (Phoenix Pharmaceuticals Inc., Burlingame, CA) was dissolved in phosphate-buffered saline (PBS, $0.01 \mathrm{M}$ ) and administered via i.p. injection at different dosages $(10,30$, and $90 \mu \mathrm{g} / \mathrm{kg}) 1 \mathrm{~h}$ after SAH according to the previous study [33]. For the long-term outcome study, an additional dose of PACAP38 was injected on days 2 and 3 after SAH.

\section{Neurobehavioral Test}

\section{Short-Term Neurological Testing}

Short-term neurological performance was evaluated using the modified Garcia test (ranging from 0 to 18 ) and beam balance test (ranging from 0 to 4 ) as previously described $[3,30]$. The modified Garcia test score was used to evaluate response capacity, alertness, coordination, and motor skills. The beam 
balance test was used to assess complex movements and coordination. A higher score represents better neurological function. Each test was examined by and independent investigator blinded to group information.

\section{Long-Term Neurological Testing}

The rotarod test was conducted on days 7,14 , and 21 post$\mathrm{SAH}$ to assess sensorimotor coordination and balance as previously described $[31,34]$. The rotating speed started with 5 revolutions per minute (RPM) or 10 RPM, and accelerated by $2 \mathrm{RPM}$ every $5 \mathrm{~s}$, and the final duration on the rotarod was recorded. The water maze tests were conducted from days 23 to 27 post-SAH to assess spatial learning capacity and memory as previously described [30]. The duration to reach the submerged platform was recorded each day. The 60 -s probe test was conducted on day 28 to record swimming distance, escape latency, and swimming path using Computer Tracking System (San Diego Instruments Inc., San Diego, CA).

\section{Intracerebral Pressure Measure}

Under anesthesia, rats were placed on prone position and the atlantooccipital membrane was exposed by a midline incision. A PE-50 catheter (Becton Dickinson, Franklin Lakes, NJ) filled with saline was gently inserted 2 to $3 \mathrm{~mm}$ depth into the cisterna magna and fixed with dental cement and cyanoacrylate glue to prevent the potential CSF leakage. The data was recorded after ICP equilibration using WINDAQ dataacquisition system (DATAQ, Akron, $\mathrm{OH}$ ).

\section{Brian Water Content}

Brain water content was evaluated via wet weight/dry weight method [35]. Under deep anesthesia, rats were decapitated, and the brains were immediately removed from skull and weighed to obtain the wet weight. They were then placed in an oven $\left(105^{\circ} \mathrm{C}\right)$ for $72 \mathrm{~h}$ to obtain the dry weight. The brain water content was calculated as follows: [(wet weight - dry weight) / (wet weight)] $\times 100 \%$.

\section{Glymphatic System Assessment (Evans Blue Cisterna Magna Injection)}

Cisterna magna injection of EB dye was conducted to observe the movement of CSF after SAH as previously described [36]. Anesthetized rats were placed in the stereotaxic frame, and the atlantooccipital membrane was exposed by a midline incision. A precision 50- $\mu$ l Hamilton syringe needle (Hamilton Company Inc., Reno, NV) was inserted 1 to $1.5 \mathrm{~mm}$ depth into the cisterna magna followed by an injection of $25 \mu \mathrm{l}$ of $2 \%$ EB dye (Sigma-Aldrich, St. Louis, MO) within $25 \mathrm{~min}$ at rate of $1 \mu \mathrm{l} / \mathrm{min}$. EB was allowed to circulate for another $35 \mathrm{~min}$ (total of $1 \mathrm{~h}$ from the start of cistern magna injection).

Serial blood samples were collected from the rat femoral vein at 0,30 , and $60 \mathrm{~min}$ after the start of EB injection. After the blood collection at $60 \mathrm{~min}$ time point, the rats received transcardiac perfusion with $100 \mathrm{ml}$ PBS $(0.01 \mathrm{M})$ before sacrifice. Brain and deep cervical lymph nodes (dcLNs) were collected after sacrifice. Three methods were used to quantify the EB dye distribution in the perivascular space and brain parenchyma [37]. The ventral surface of the rat brain was divided into six different segments along the arteries (R1R6). The concentration of EB dye in the forebrain parenchyma (R1-R3), the number of EB dye positive sections, and EB dye pixel area were quantified (Fig. 4d).

In addition, the forebrain and dcLNs were homogenized in $50 \%$ trichloroacetic acid (TCA, Sigma-Aldrich, St. Louis, $\mathrm{MO})$ to measure the EB concentration. The blood samples were also mixed with equal volume TCA to measure EB concentration. All samples with TCA were incubated at $4{ }^{\circ} \mathrm{C}$ overnight. Then, the sample was centrifuged at 15,000 revolutions per minute (RPM) in $4{ }^{\circ} \mathrm{C}$ for $30 \mathrm{~min}$. The supernatant was measured using a spectrophotometer (Thermo Spectronic Genesys 10 UV, Thermo Fischer Scientific Inc., Waltham, MA) at $610 \mathrm{~nm}$ and quantified with a standard curve.

\section{BBB Permeability Assessment (Evans Blue Intraperitoneal Injection for Extravasation Assay)}

EB extravasation assay was conducted to evaluate BBB permeability $[3,38]$. First, a $4 \%$ EB solution $(2 \mathrm{ml} / \mathrm{kg})$ was injected i.p. at $22 \mathrm{~h}$ after SAH, followed by $2 \mathrm{~h}$ of circulation. The rats were sacrificed at $24 \mathrm{~h}$ after SAH. The brain samples collection and EB concentrations measurements were conducted as described above.

\section{Histological Staining}

Histological staining of brain sections was prepared as previously described [31]. Under deep anesthesia, rats were transcardiac perfusion using a $0.01 \mathrm{M}$ PBS and $10 \%$ neutral buffered formalin. Brains were fixed with $10 \%$ neutral buffered formalin for $24 \mathrm{~h}$ and then in $4 \%$ paraformaldehyde for $24 \mathrm{~h}$. Next, they were dehydrated in serial 15 and $30 \%$ sucrose solutions for 2 days. The brain was then cut into coronal frozen slices, $\sim 10$ to $15 \mu \mathrm{m}$ thick, and fixed on the slide for histological staining, including double immunofluorescence, immunohistochemical staining, and Nissl staining. Images were visualized and photographed using a fluorescence microscope (Leica Microsystems, Wetzlar, Germany) and optical microscope (Olympus, Center Valley, PA). 


\section{Double Immunofluorescence}

Briefly, brain slices $(10 \mu \mathrm{m})$ were incubated with $5 \%$ normal donkey serum and $0.1 \%$ Triton $\mathrm{X}-100$ for $1 \mathrm{~h}$ at room temperature, followed by overnight incubation with the primary antibody at $4{ }^{\circ} \mathrm{C}$. The primary antibodies are listed as follows: anti-PAC1 (A13859, Abclonal, Woburn, MA), anti-VPAC1 (LS-C386658, LSbio, Seattle, WA), anti-VPAC2 (LSC 386653 , L Sbio), anit-SUR 1 (ab134292, Abcam, Cambridge, MA), anti-AQP4 (SC-32739, Santa Cruz Biotechnology), anti-NeuN (marker of neuron, ab104224, Abcam), anti-GFAP (marker of astrocyte, ab53554, Abcam), and anti-von Willebrand factor (vWF) (marker of endothelia cell, SC-365712, Santa Cruz Biotechnology). Then, the slices were incubated with species-corresponding fluorescence-conjugated secondary antibodies (Jackson ImmunoResearch, West Grove, PA) for $1 \mathrm{~h}$ at room temperature.

\section{Immunohistochemical Staining of IgG}

IgG staining was conducted to evaluate the BBB permeability, according to the protocol of VECTASTAIN ABC Kit (PK4004, VECTOR Laboratories, Burlingame, CA). Briefly, brain slices (10 $\mu \mathrm{m}$ thick) were incubated with serial solutions at room temperature: $0.1 \%$ Triton $\mathrm{X}-100$ for $1 \mathrm{~h}, 1 \%$ hydrogen peroxide $\left(\mathrm{H}_{2} \mathrm{O}_{2}\right)$ for $15 \mathrm{~min}$ and $10 \%$ rabbit serum for $1 \mathrm{~h}$. Next, the slices were incubated with biotinylated anti-mouse polyclonal $\operatorname{IgG}(1: 100)$ at $4{ }^{\circ} \mathrm{C}$ overnight, followed with 1 -h incubation of avidin-biotin-horseradish peroxidase complex at room temperature. Color reactions were developed in diaminobenzidine $/ \mathrm{H}_{2} \mathrm{O}_{2}$ solution, and the sections were lightly counterstained with hematoxylin. Four continuous images of the left temporal cortex were photographed. The relative staining intensity of extravasated $\mathrm{IgG}$ was quantified using the ImageJ software (ImageJ, version 15.1, NIH, Bethesda, MD).

\section{Nissl Staining}

Brain slices (15 $\mu \mathrm{m}$ thick) were submerged in $0.5 \%$ cresyl violet solution for $10 \mathrm{~min}$ until the desired intensity of staining was achieved. Cortical thickness and ventricular area were analyzed by the ImageJ software. Ventricular volume was measured according to a previous study [39] and was determined by calculating the average of ventricular area at various levels of the preoptic chiasm, fornix-hippocampus commissure, and rear hippocampus, which was then multiplied with total section thickness. Hippocampal neuronal loss was evaluated as previously described [40]. Microphotographs of three ipsilateral (left) hippocampal regions including cornu ammonis (CA)1, CA3, and dentate gyrus (DG) were taken at $\times 20$ magnification for 3 continuous brain slices/rat. The number of Nissl-positive neurons was calculated using the
ImageJ software. The data was expressed as the number of neurons per field.

\section{Western Blot}

Western blot was performed as previously described [31]. The ipsilateral (left) cerebral hemisphere were homogenized in RIPA lysis buffer (Santa Cruz Biotechnology). The supernatant was mixed with loading buffer until a concentration of $5 \mu \mathrm{g} / \mathrm{mL}$ was reached. Equal amounts of protein samples $(20 \mu \mathrm{g})$ were loaded onto SDS- PAGE gels (7.5-12\%), followed by electrophoresis, and then transferred onto the nitrocellulose membrane $(0.22 \mu \mathrm{m})$. The membrane was incubated with blocking buffer ( $5 \%$ no-fat milk) for $1 \mathrm{~h}$ at room temperature, followed with overnight incubation with the primary antibody at $4{ }^{\circ} \mathrm{C}$. The primary antibodies included the following: anti-PACAP (ab181205, Abcam), anti-PAC1 (A13859, Abclonal), anti-VPAC1 (LS-C386658, LSbio), anti-VPAC2 (LS-C386653, LSbio), anti-SUR1 (ab134292, Abcam), anti-AQP4 (sc-32739, Santa Cruz Biotechnology), anti-cAMP (ab76238, Abcam), anti-PKA (4781S, Cell Signaling Technology, Danvers, MA), anti-p-PKA (4782S, Cell Signaling Technology), anti-MMP-9 (ab38898, Abcam), anti-ZO-1 (sc-33725, Santa Cruz Biotechnology), and anti- $\beta$-actin (sc-47778, Santa Cruz Biotechnology). On the second day, the species-corresponding secondary antibody (Santa Cruz Biotechnology) was incubated with the membrane at room temperature for $1 \mathrm{~h}$. Next, the immunoblots were visualized using the ECL Plus chemiluminescence reagent kit (Amersham Bioscience, Pittsburgh, PA), and quantified by the ImageJ software.

\section{Immunoprecipitation}

All immunoprecipitations were conducted according to the manufacturer's instructions (Abcam). Briefly, either $2 \mu \mathrm{g}$ anti-AQP4 antibody (sc-32739; Santa Cruz Biotechnology), anti-Phospho-(Ser/Thr) Phe antibody (\#9631, Cell Signaling Technology), or normal mouse IgG (Millipore, Burlington, MA) was added to $200 \mu \mathrm{g}$ of the total proteins that were prepared. Then, $50 \mu \mathrm{l}$ of protein A sepharose beads (ab193262, Abcam) were added to each reaction mix and incubated overnight at $4{ }^{\circ} \mathrm{C}$. On the second day, the beads were washed 4 times in $500 \mu$ l of PBS, harvested via centrifugation, resuspended in $30 \mu$ lof loading buffer, and heated at $95{ }^{\circ} \mathrm{C}$ for $3 \mathrm{~min}$ prior to western blot. The western blot was conducted as previously described.

\section{Statistical Analysis}

All data were presented as the mean and standard deviation (mean \pm SD). One-way ANOVA, followed by Tukey's post hoc test, was used to compare multiple groups. Two-way 
ANOVA followed by Tukey's post hoc test was used to compare the changes according to the different levels of multiple categorical variables (e.g., brain water content comparison of different hemispheres in different groups). The KruskalWallis test was used to compare the difference of data in abnormal distribution. Statistical analysis was conducted using GraphPad Prism 8.2.1 (GraphPad Software, San Diego, CA) and SPSS 23.0 (IBM, Armonk, NY). $P<0.05$ was considered statistically significant.

\section{Results}

\section{Animal Use and Mortality}

A total of 334 male SD rats were used, with 54 and 280 undergoing sham surgery and SAH surgery, respectively. Of $280 \mathrm{SAH}$ rats, 16 rats were excluded due to mild SAH. The total mortality of SAH group was $22.7 \%$ (60/264). Mortality details in each group was listed in the Supplementary Table.

\section{Endogenous PACAP Knockout Exacerbated Short- Term Outcomes After SAH}

For validation of PACAP knockout CRISPR, the western blot showed PACAP knockout CRISPR significantly decreased PACAP protein expression at $24 \mathrm{~h}$ after SAH compared with SAH group $(P=0.035)$ and SAH + control CRISPR group $(P=0.024)$ (Fig. $2 \mathrm{~g}, \mathrm{~h})$. Besides, SAH + PACAP control CRISPR showed no statistical difference compared with $\mathrm{SAH}$ group in either SAH grade or neurological scores or brain water content or ICP or PACAP expression (both $P>0.999$ ) (Fig. 2b-h).

There was no statistical difference of SAH grade between SAH groups $(P>0.999$; Fig. $2 b)$. ICP and brain water contents were used for assessing brain edema. Compared with shams, the ICP and bilateral hemispheres brain water content were significantly increased $24 \mathrm{~h}$ after SAH (both $P<0.001$ compared with the SAH group) (Fig. 2a, e, f). The PACAP knockout CRISPR significantly exacerbated the elevation of ICP $(P=0.014)$ and brain water content $24 \mathrm{~h}$ after SAH $(P<0.001)$, compared with the SAH group. Consistently, SAH rats were associated with lower neurological scores than shams (both $P<0.001$ ). The modified Garcia score was further decreased by knockout of endogenous PACAP, compared with the SAH group $(P=0.032)$.

In order to explore the changes of edema-related proteins after the PACAP knockout-induced brain edema, the western blot results revealed that PACAP knockout significantly aggravated the increases in edema-related proteins levels of AQP4 $(P=0.047)$ and SUR1 $(P=0.039)$ in ipsilateral hemisphere SAH $24 \mathrm{~h}$ after SAH (Fig. 2g, h).

\section{Time Course of Endogenous PACAP, PACAP Receptors, and SUR1 Expressions in the Ipsilateral Hemisphere After SAH}

The endogenous PACAP protein levels significantly increased from 3 to $72 \mathrm{~h}$ after SAH, peaking at $24 \mathrm{~h}$, compared with the sham group (both $P<0.027$; Fig. 3a, b). Similarly, the PAC1 receptor, significantly increased from 12 to $72 \mathrm{~h}$ after SAH, peaking at $24 \mathrm{~h}$, compared with the sham group (both $P<0.013$; Fig. 3a, b). However, receptors of VPAC1 $(P>0.523)$ and VPAC2 $(P>0.456)$ did not changes over time after SAH when compared with the sham group (Fig. 3a, b). Compared with the sham group, the SUR1 protein levels started increasing at $3 \mathrm{~h}$ after SAH and peaked at $24 \mathrm{~h}$ (both $P<0.010$; Fig. 3a, b).

The double immunofluorescence staining was conducted at $24 \mathrm{~h}$ after SAH because of the peak protein expressions at this time point. As shown in Fig. 3c, g, although PAC1 was expressed on neurons in the sham and SAH groups, the expressions of $\mathrm{PACl}$ were increased on astrocytes and endothelial cells in SAH group $(P<0.001)$. VPAC1 was mainly expressed on astrocytes, but rarely on neurons and endothelial cells in both sham and SAH groups (Fig. 3d). VPAC2 was mainly expressed on neurons, but rarely on astrocytes and endothelial cells in both sham and SAH groups (Fig. 3e). Both VPAC1 and VPAC2 presented no change after SAH (Fig. 3g). The SUR1 was barely expressed in the brains of shams, but the expressions were significantly increased on neurons, astrocytes, and endothelial cells after SAH (both $P<0.001$; Fig. 3f, g).

\section{Exogenous PACAP38 Treatment Attenuated Brain Edema and Neurological Deficits at $24 \mathrm{~h}$ After SAH}

There was no significant difference regarding SAH score among SAH groups $(P>0.999$; Fig. 4a). ICP was significantly increased $24 \mathrm{~h}$ after SAH when compared with the sham group $(P<0.001$; Fig. 4d). PACAP38 treatments, at doses of $30 \mu \mathrm{g} / \mathrm{kg}$ and $90 \mu \mathrm{g} / \mathrm{kg}$, significantly decreased ICP at $24 \mathrm{~h}$ after SAH when compared with the SAH + PBS group (both $P<0.001$; Fig. $4 d$ ). Similarly, the brain water content was significantly increased in the bilateral hemispheres and cerebellum at $24 \mathrm{~h}$ after SAH when compared with the sham group (both $P<0.001$; Fig. 4e). PACAP38 treatments at doses of $30 \mu \mathrm{g} / \mathrm{kg}$ and $90 \mu \mathrm{g} / \mathrm{kg}$, significantly decreased the brain water content in the ipsilateral (left) hemisphere $(P=0.001$ and $P<0.001$ respectively). PACAP38 at dose of $30 \mu \mathrm{g} / \mathrm{kg}$ also decreased brain water content in the contralateral (right) hemisphere when compared with the SAH + PBS group $(P=$ 0.007; Fig. 4e).

The modified Garcia scores and the beam balance test score were significantly decreased $24 \mathrm{~h}$ after SAH when compared with the sham group $(P=0.003$ and $P=0.011$ respectively; 
a
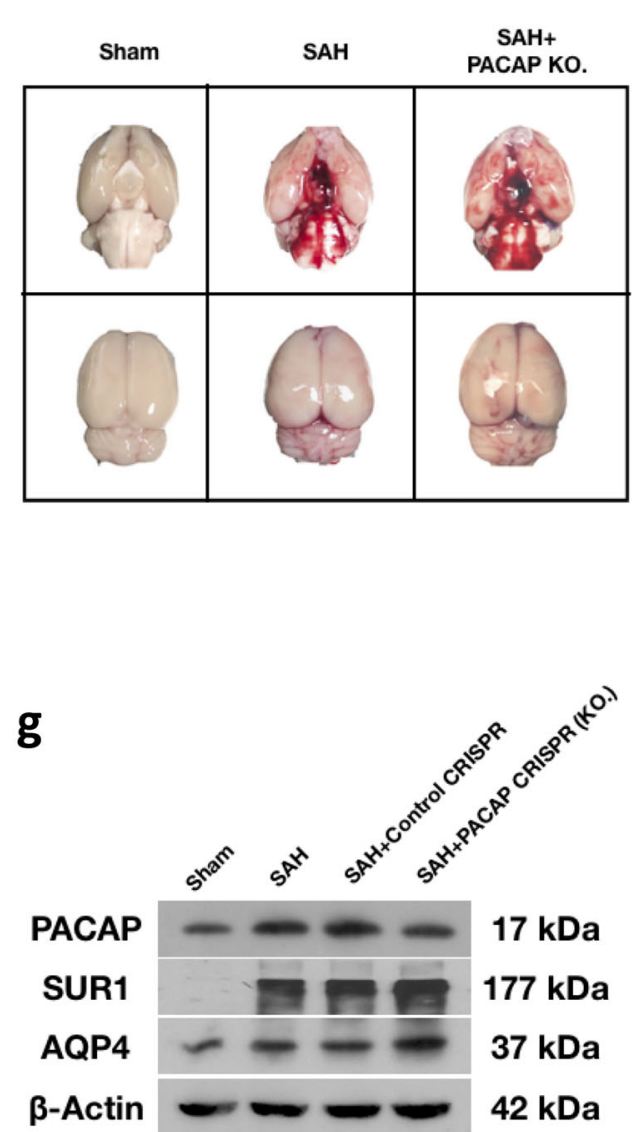

b

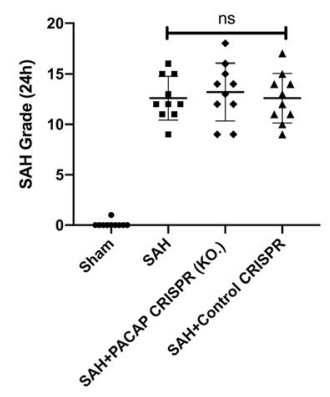

C

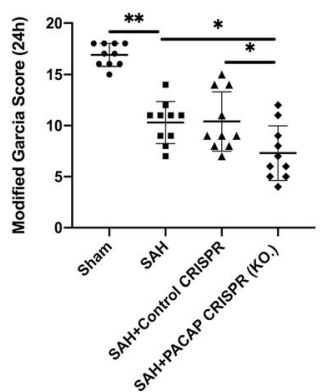

d

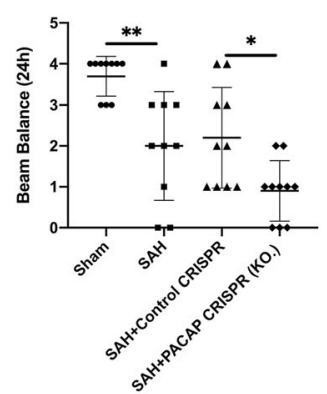

e

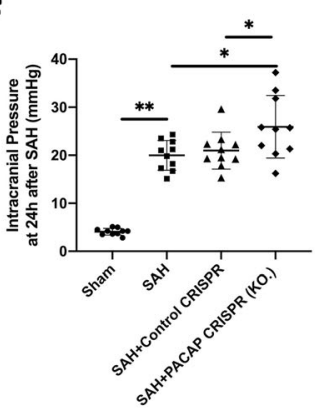

f

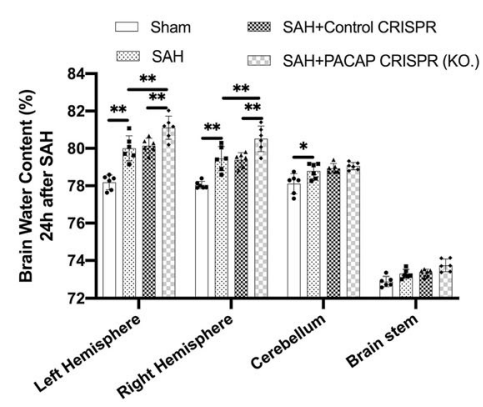

h
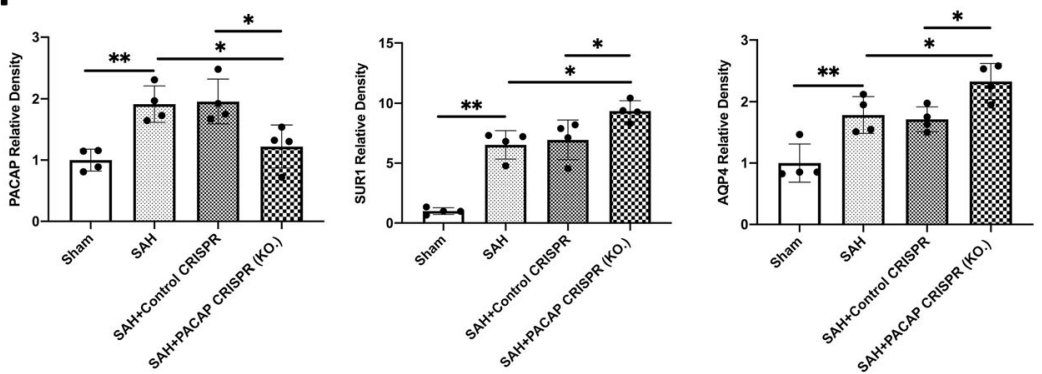

Fig. 2 Endogenous PACAP knockout aggravated brain edema and neurological deficits at $24 \mathrm{~h}$ after SAH. (a) Representative image of brain outline of sham, SAH, SAH + PACAP knockout CRISPR; (b) SAH grade; (c, d) PACAP knockout CRISPR significantly aggravated decrease of modified Garcia score and beam balance score after SAH; (e) PACAP knockout CRISPR significantly aggravated ICP elevation after

SAH; (f) PACAP knockout CRISPR significantly aggravated brain water content increasement in bilateral hemisphere after SAH; (g) representative western blot bands of PACAP, SUR1, and AQP4; (h) densitometric quantification of PACAP, SUR1, and AQP4 at $24 \mathrm{~h}$ after SAH. Data was represented as mean \pm SD. $n=4-10$ /group. $* P<0.05$, $* * P<0.01$; ns $=$ not statistically significant. One-way ANOVA, Tukey's post hoc test

Fig. $4 \mathrm{~b}, \mathrm{c})$. PACAP38 treatment, at a dosage of $30 \mu \mathrm{g} / \mathrm{kg}$ significantly improved both modified Garcia score and beam balance score at $24 \mathrm{~h}$ after SAH $(P=0.003$ and $P=0.023$ respectively; Fig. 4b, c).

Based on the short-term outcomes results, the dose of $30 \mu \mathrm{g} / \mathrm{kg}$ was determined to be the best dosage of PACAP38 treatment for the following experiments.

\section{PAC1 Receptor Mediated the Neuroprotection of Exogenous PACAP38 Treatment}

There were no significant differences in SAH scores between SAH groups $(P=0.980$; Fig. $4 \mathrm{~g})$. The pretreatment with the PAC1 selective inhibitor, PACAP6-38 significantly abolished the anti-edema effects and neurologic function benefits of PACAP38 in SAH rats when compared with the $\mathrm{SAH}+\mathrm{PACAP} 38+\mathrm{DMSO}$ group $(P=0.048$ for modified Garcia score, $P=0.027$ for beam balance score, $P=0.028$ for ICP, $P<0.009$ for brain water content; Fig. $4 \mathrm{~h}-\mathrm{j}$ ). However, the pretreatment of VPAC1/2 inhibitor did not affect the neuroprotective effects of PACAP38 treatment in SAH rats when compared with the SAH + PACAP38 + DMSO group ( $P>0.999$; Fig. 4h-j).

\section{Exogenous PACAP38 Treatment Attenuated BBB Disruption at $24 \mathrm{~h}$ After SAH}

IgG staining and EB dye extravasation were used for assessing BBB disruption. As shown in Fig. 5a, SAH significantly increased $\mathrm{IgG}$ extravasation in the ipsilateral 
a

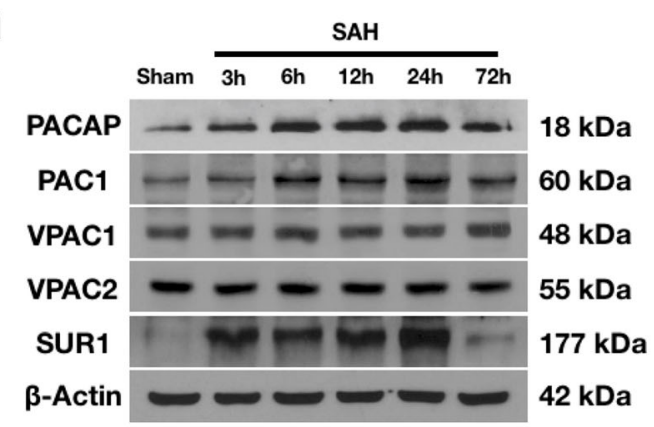

b
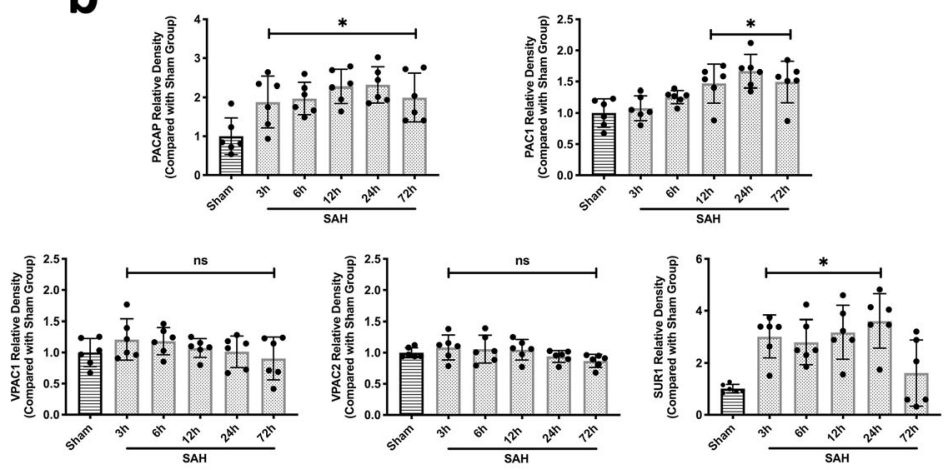

C
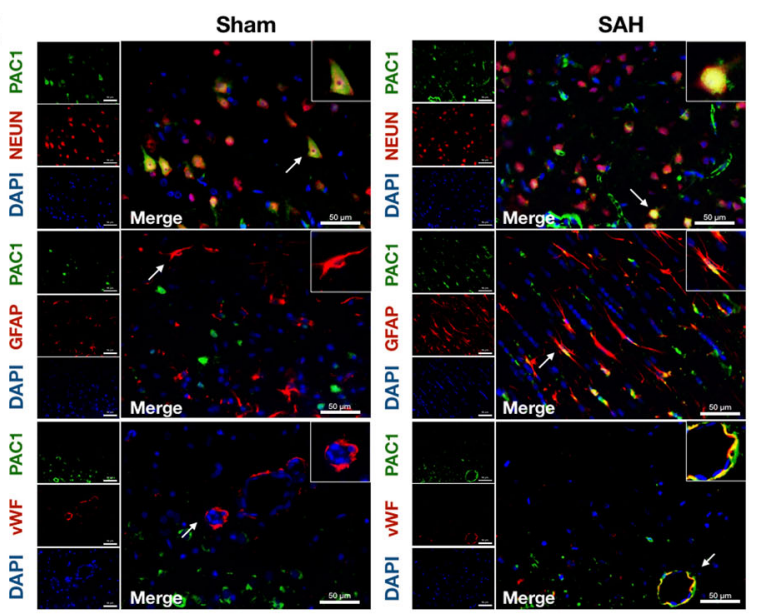

e
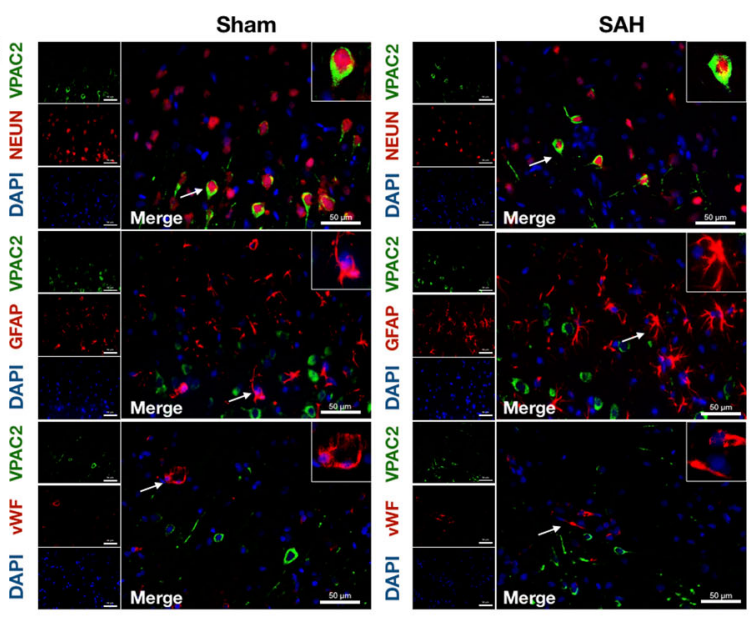

g
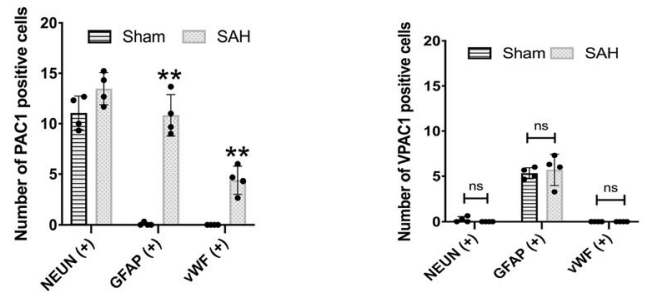

d

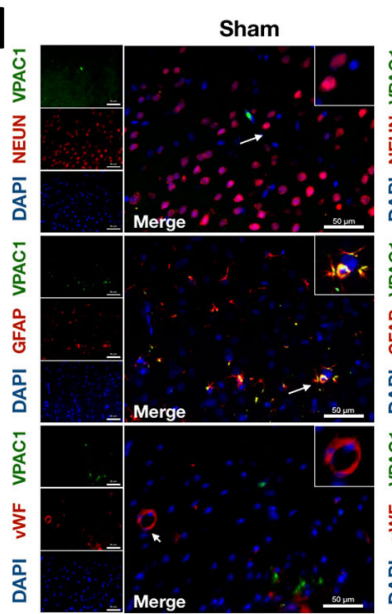

f

Sham
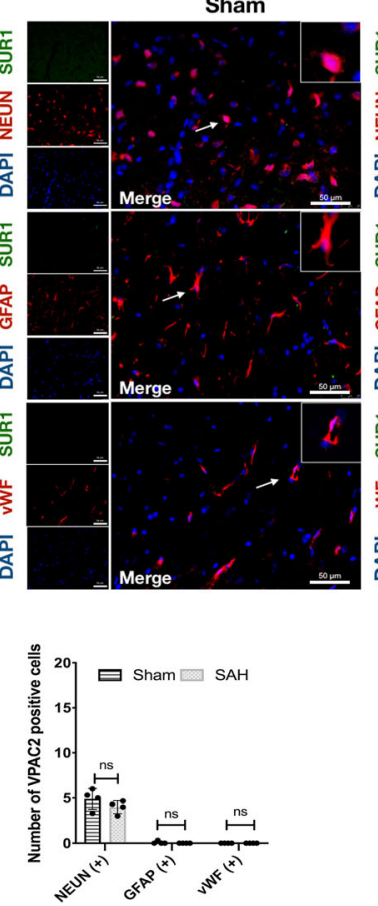

SAH

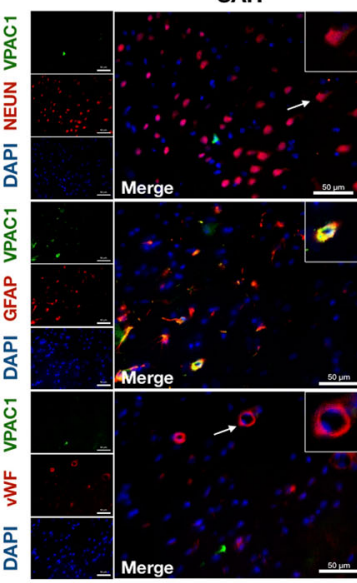

SAH

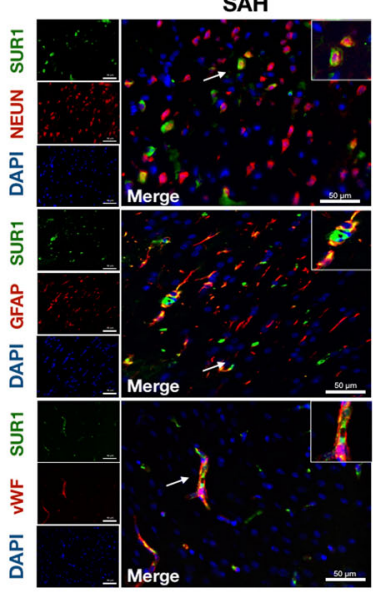

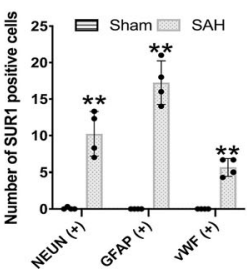


Fig. 3 Expression level of endogenous PACAP, PAC1, VPAC1, VPAC2, and SUR1 in the ipsilateral hemisphere after SAH. (a) Representative western blot bands of each protein; (b) densitometric quantification of endogenous PACAP, PAC1, VPAC1, VPAC2, and SUR1, $n=6$ per group; (c-f) representative microphotographs of coimmunofluorescence staining of PAC1, VPAC1, VPAC2, and SUR1 (green) with neurons (NeuN, red), astrocytes (GFAP, red), and endothelial cells (vWF, red) in the ipsilateral basal cortex of sham and SAH $(24 \mathrm{~h})$ group; (g) quantification of PAC1, VPAC1, VPAC2, and SUR1-positive cells. Positive cells were counted in three randomized areas chosen in the ipsilateral cortex for imaging. $n=4$ per group. Scale bar $=50 \mu \mathrm{m}$. Data was represented as mean $\pm \mathrm{SD} . * P<0.05$ versus sham group, $* * P<0.01$ versus sham group; $\mathrm{ns}=$ not statistically significant. One-way ANOVA, Tukey's post hoc test for (b); two-way ANOVA, Tukey's post hoc test for (g)

hemisphere at $24 \mathrm{~h}(P<0.001)$, suggesting increases in BBB permeability. PACAP38 treatment significantly attenuated SAH-induced IgG extravasation $(P<0.001)$. The results were

a

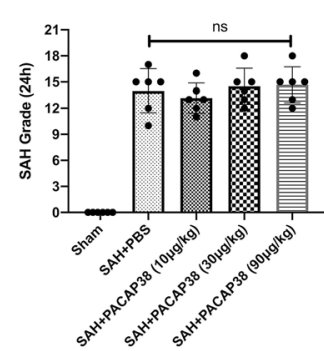

b

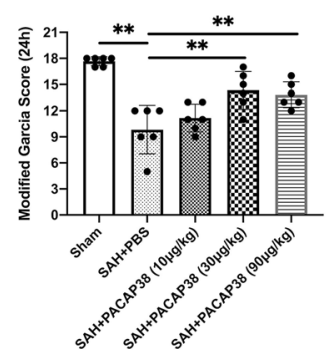

e

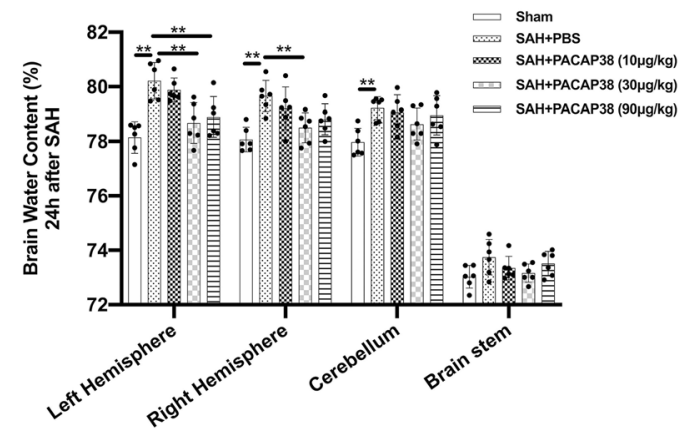

g

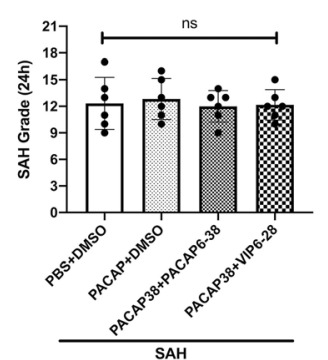

h

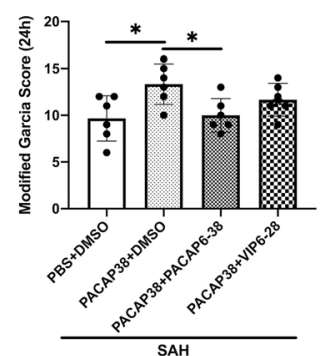

Fig. 4 Exogenous PACAP38 treatment attenuated brain edema and neurological deficits at $24 \mathrm{~h}$ after SAH. (a) SAH grade; (b, c) exogenous PACAP38 $30 \mu \mathrm{g} / \mathrm{kg}$ significantly improved modified Garcia score and beam balance score at $24 \mathrm{~h}$ after SAH. PACAP38 $90 \mu \mathrm{g} / \mathrm{kg}$ also significantly improved modified Garcia score at $24 \mathrm{~h}$ after SAH; (d, e) PACAP38 $30 \mu \mathrm{g} / \mathrm{kg}$ and $90 \mu \mathrm{g} / \mathrm{kg}$ significantly decreased ICP and brain water content in left hemisphere at $24 \mathrm{~h}$ after SAH. PACAP38 $30 \mu \mathrm{g} / \mathrm{kg}$ also decreased brain water content in right hemisphere; (f) PAC1 consistent with the EB dye extravasation assay, in which PACAP38 also significantly reduced the SAH-induced EB dye extravasation in the ipsilateral hemisphere $24 \mathrm{~h}$ after SAH (both $P<0.001$; Fig. 5b). The SAH grades were comparable among all SAH groups $(P=0.950$ and 0.901 , respectively) (Supplementary Fig. 1).

\section{Exogenous PACAP38 Treatment Attenuated Glymphatic System Dysfunction at 24 h After SAH}

There was no significant difference in SAH score among $\mathrm{SAH}$ groups $(P=0.970$; Supplementary Fig. 1$)$. At $1 \mathrm{~h}$ after cisterna magna injection, EB dye was grossly visible on the brain surface (subarachnoid space) and the perivascular space near the Willis cycle and the belonging arteries in the sham group. The spread of EB dye was impaired in the SAH + PBS group,
C

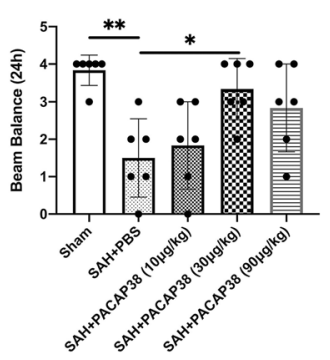

d

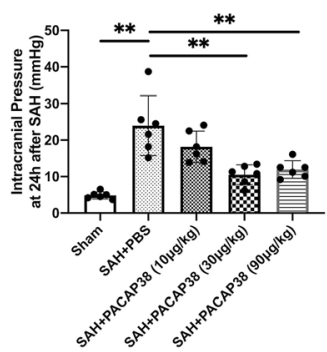

$\mathbf{f}$

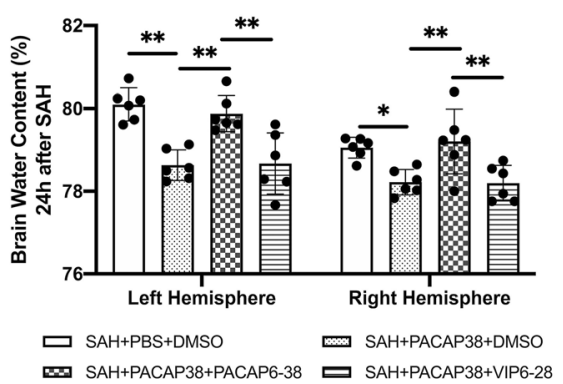

i
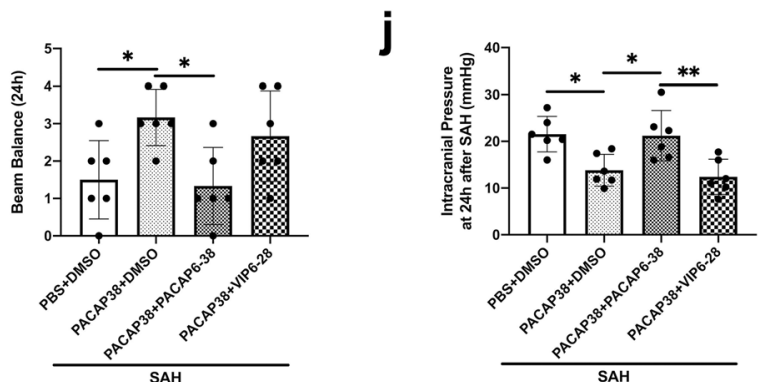

inhibitor, PACAP6-38 abolished PACAP38-induced alleviation of brain water content after SAH; (g) SAH grade; (h, i) PACAP6-38 abolished PACAP38-induced neurobehavior improving after SAH, with VPAC1/2 inhibitor, VIP6-28 showed no effect; (j) PACAP6-38 abolished PACAP38-induced alleviation of ICP after SAH. $n=6 /$ group. $* P<0.05, * * P<0.01 ; \mathrm{ns}=$ no statistical significance. One-way ANOVA, Tukey's post hoc test for $(\mathrm{a}-\mathrm{d})$ and $(\mathrm{g}-\mathrm{j})$; two-way ANOVA, Tukey's post hoc test for $(\mathrm{e}, \mathrm{f})$ 


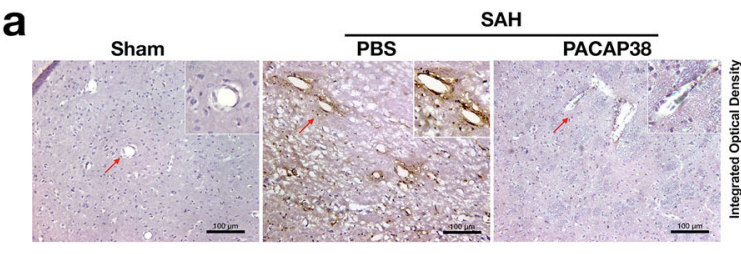

C

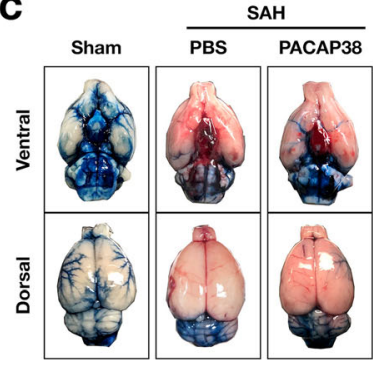

d

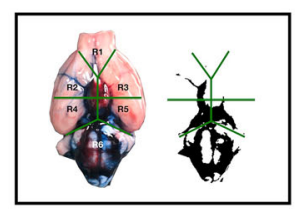

e

\section{$\mathbf{f}$}
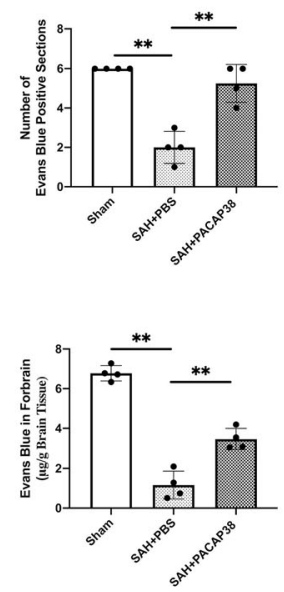

b

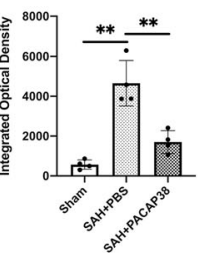

SAH

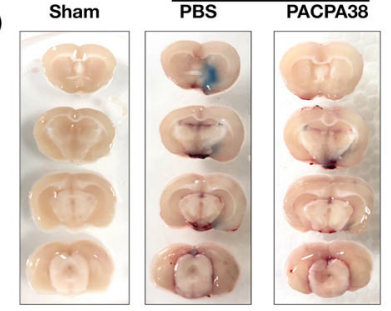

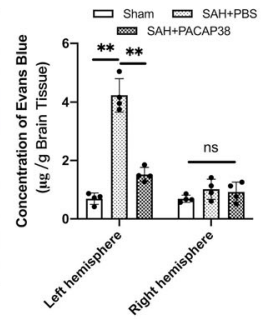

g

h
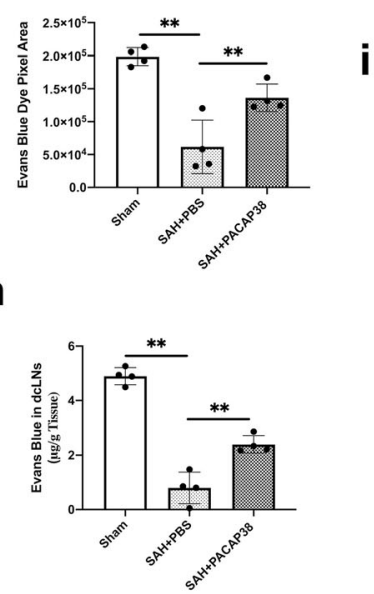

(1)

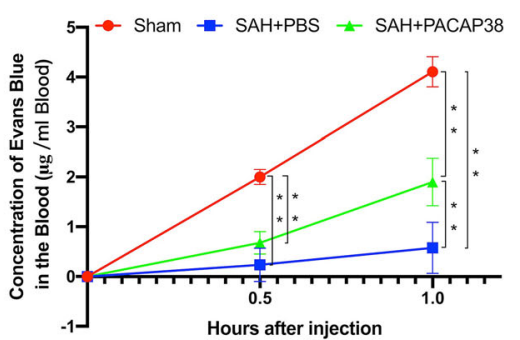

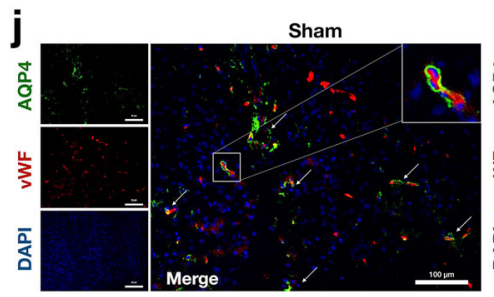
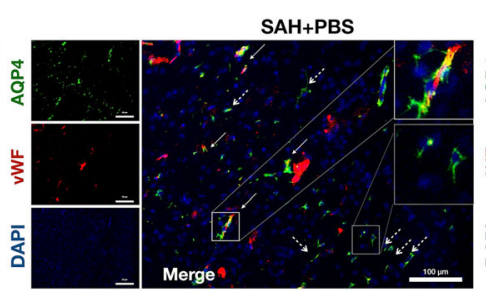
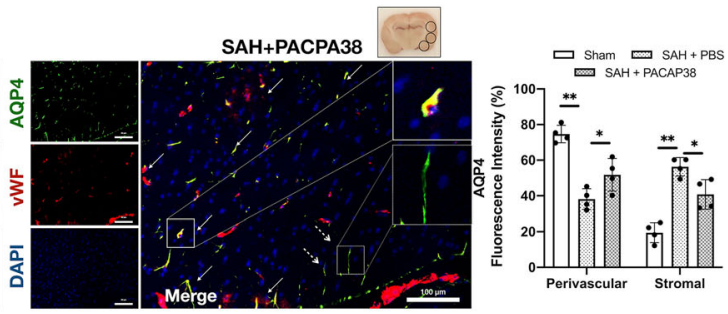

Fig. 5 Exogenous PACAP38 treatment attenuated BBB disruption and glymphatic dysfunction $24 \mathrm{~h}$ after SAH. Exogenous PACAP38 markedly reduced IgG and Evans blue extravasation, as well as prevented CSF movement/clearance disorder and AQP4 depolarization $24 \mathrm{~h}$ after SAH. (a) Representative microphotographs and quantification of positive area optical density of IgG staining. Data was measured through three randomized areas chosen in the ipsilateral cortex for imaging and averaged for each animal. Scale bar $=100 \mu \mathrm{m}$. (b) Representative image of Evans blue extravasation and concentration of Evans blue ( $\mu \mathrm{g} / \mathrm{g}$ brain tissue) in the left and right hemisphere in each group. Rats received i.p. Evans blue (4\%, $2 \mathrm{ml} / \mathrm{kg}$ ) injections $2 \mathrm{~h}$ before sacrificed. (c) Representative image of Evans blue distribution in subarachnoid space $1 \mathrm{~h}$ after Evans blue (2\%) injection into cisterna magna. (d) Six segments divided on ventral brain. (e) Evans blue positive section on the ventral brain. (f) Concentration of

but was improved by PACAP38 treatment (Fig. 5c). Three methods were used to quantify the EB dye distribution in the perivascular space and brain parenchyma. The ventral surface of the rat brain was divided into six different segments along the arteries (R1-R6; Fig. 5d). The concentration of EB dye in the forebrain parenchyma (R1-R3), the number of EB dyepositive sections, and EB dye pixel area were analyzed.

Three quantification methods consistently demonstrated there were the stagnation of brain distribution of EB dye after
Evans blue ( $\mu \mathrm{g} / \mathrm{g}$ brain tissue) in forebrain. (g) Evans blue dye pixel area. (h) Concentration of Evans blue ( $\mu \mathrm{g} / \mathrm{g}$ tissue) in dcLNs. (i) Blood Evans blue concentration $0.5 \mathrm{~h}$ and $1 \mathrm{~h}$ after cisterna magna injection. (j) Representative microphotographs of co-immunofluorescence staining and quantitation of perivascular and stromal AQP4 fluorescence intensity percent in the ipsilateral basal cortex at $24 \mathrm{~h}$. Green represented AQP4 with red represented endothelial cells (vWF). Solid lines/arrows indicate perivascular AQP4 and dash lines/arrows indicate stromal AQP4. Scale bar $=100 \mu \mathrm{m}$. Fluorescence was measured in three randomized areas chosen in the ipsilateral cortex for imaging. Perivascular and stromal fluorescence intensity percent were averaged for each animal. $n=4$ / group. $* P<0.05, * * P<0.01 ; \mathrm{ns}=$ no significance. One-way ANOVA, Tukey's post hoc test for (a-h); two-way ANOVA, Tukey's post hoc test for $(i, j)$

SAH when compared with the sham group (both $P<0.001$ ), evidenced by significantly decreases in the number of EBpositive section (Fig. 5e), EB concentration in forebrain (Fig. 5f), and EB dye pixel area (Fig. 5g). PACAP38 treatment significantly improved such impairment of CSF movement when compared with the SAH + PBS group (both $P<0.008$; Fig. $5 \mathrm{e}-\mathrm{g})$.

Furthermore, the dcLNs EB concentration suggested that SAH significantly impaired the clearance of EB dye from the 
subarachnoid space to dcLNs $1 \mathrm{~h}$ after EB dye cisterna injection $(P<0.001$ compared with the sham group; Fig. 5 h). Meanwhile, plasma EB dye concentrations also suggested that SAH significantly impaired the clearance capacity at $30 \mathrm{~min}$ and $1 \mathrm{~h}$ after EB dye cisterna injection $(P<0.001$ compared with the sham group; Fig. 5i). However, both the PACAP38 treatment significantly accelerated the clearance of EB dye from the CSF to dcLNS $(P=0.001)$ and blood $(P<0.001)$ at $1 \mathrm{~h}$ after EB injection, compared with the SAH + PBS group (Fig. 5h, i).

The immunofluorescence was conducted to evaluate the change of perivascular and stromal AQP4 expressions. The results showed that SAH largely reduced the perivascular localization of AQP4 $(P<0.001)$ but increased the stromal AQP4 expression $(P<0.001$; Fig. 5i). PACAP38 treatment promoted the perivascular AQP4 polarization $24 \mathrm{~h}$ after SAH $(P=0.026$ of perivascular AQP4, $P=0.011$ of stromal AQP4; Fig. 5i).

\section{Exogenous PACAP38 Treatment Improved Long-Term Neurological Performance at 28 Days After SAH}

SAH markedly impaired the rotarod test performance at both 5 and 10 RPM acceleration velocity compared with the sham group (both $P<0.001$; Fig. 6a, b). PACAP38 treatment significantly increased the falling latency of both acceleration velocity at the first 2 weeks (5 RPM: $P=0.029$ and 0.026 , respectively; 10 RPM: $P=0.019$ and 0.019 , respectively) and that of 5 RPM acceleration velocity at the third week, when compared with the SAH + PBS group $(P=0.026$; Fig. $6 a, b)$.

In the Morris water maze test, SAH impaired the spatial memory and learning ability with a longer escape latency and swimming distance to the platform than shams on days 24 to 27 after SAH (both $P<0.001$; Fig. 6c, d). PACAP38 treatment significantly shortened escape latency on days 25 to 27 after SAH $(P=0.014,0.046$, and 0.012 respectively), with reduced swimming distance on days 25 to 26 after SAH ( $P=0.024$ and 0.044 respectively), compared with the SAH + PBS group (Fig. 6c, d). In the probe trials, the rats in the $\mathrm{SAH}+\mathrm{PBS}$ group stayed significantly shorter probe quadrant duration $(P=0.003)$ and swimming speed $(P=0.028)$, compared with sham (Fig. 6e, f). PACAP38 treatment significantly increased the quadrant duration $(P=0.012$; Fig. 6 e, f), but not swimming speed $(P=0.063$; Fig. 6e, g) compared with the $\mathrm{SAH}+\mathrm{PBS}$ group.

\section{Exogenous PACAP38 Treatment Reduced Hydrocephalus and Neuronal Degeneration at 28 Days After SAH}

Nissl staining was used to analyze the morphology of the lateral ventricular volume and hippocampal neuronal loss.
The SAH-induced bilateral ventricular dilation and cortical thickness reduction in the SAH + PBS group was significantly improved than that in the PACAP38-treated group $(P=0.005$ and $P=0.047$, respectively; Fig. $6 \mathrm{~h}-\mathrm{j}$ ). In ipsilateral hippocampus, PACAP38 treatment significantly reduced SAHinduced neuron loss at CA1, CA3, and DG regions compared with the SAH + vehicle group $(P=0.042,0.002$, and 0.001 respectively) (Fig. 6k, 1).

\section{PACAP Provided Neuroprotection Through PAC1/AC/CAMP/PKA Pathway}

As determined by western blot, there was significant higher levels of PACAP $(P<0.001)$, PAC1 $(P=0.041)$, $\operatorname{cAMP}(P=$ $0.001)$, and p-PKA $(P=0.014)$ after SAH than shams, which were further increased by PACAP38 treatment $(P=0.011$, $0.020,0.021$, and 0.005 , respectively; Fig. 7a, b), although pretreatment with the PAC1 inhibitor PACAP6-38 significantly reversed the effects of PACAP3 8 treatment on proteins level of PAC1 $(P=0.043)$, cAMP $(P=0.034)$, and p-PKA $(P=0.013)$ (Fig. 7a, b). Pretreatment with AC inhibitor SQ22536, markedly reversed the effects of PACAP38 treatment on proteins levels of cAMP $(P<0.001)$ and p-PKA $(\mathrm{P}<$ 0.001) levels (Fig. 7a, b).

As for the BBB function-related proteins, the results showed that $\mathrm{SAH}$ marked increased proteolytic enzyme MMP-9 expression $(P<0.001)$, and tight junction protein ZO-1 degradation $(P=0.013)$. However, PACAP38 treatment significantly reversed the SAH-induced MMP-9 activation $(P<0.001)$ and ZO-1 degradation $(P=0.003$; Fig. $7 \mathrm{a}, \mathrm{b})$. Furthermore, pretreatment with PACAP6-38 and SQ22536 dramatically reversed these alterations of MMP-9 and ZO-1 when compared with the SAH + PACAP38 + DMSO group (PACAP6-38: $P=0.007$ and 0.035, respectively; SQ-22536: $P=0.035$ and 0.024 ; Fig. $7 \mathrm{a}, \mathrm{b})$.

Moreover, the expressions of AQP4 and SUR1 were increased after SAH compared with the sham group (both $P<0.001$; Fig. 7a-d). PACAP38 treatment attenuated expression of both AQP4 and SUR1 compared with the SAH + PBS + DMSO group $(P=0.004$ and $P=0.044$; Fig. $7 \mathrm{a}-\mathrm{d})$. Similarly, pretreatment with the PACAP6-38 and SQ22536 reversed the effects of PACAP38 treatment on AQP4 and SUR1 (PACAP6-38: $P=0.040$ and 0.004, respectively; SQ22536: $P=0.028$ and 0.031 , respectively) (Fig. 7a-d).

Co-IP was conducted to further investigate the relationships between AQP4 and SUR1, as well as p-PKA and SUR1. The results suggested that AQP4 co-assembled with SUR1 after SAH $(P<0.001)$, but PACAP38 abolished this interaction $(P=0.017$; Fig. $7 \mathrm{c}, \mathrm{d})$. Meanwhile, the SUR1 immunoprecipitated with a phospho-antibody, and p-SUR1 was clearly detectable in the PACAP38-treated SAH rats, but not in DMSO-treated SAH rats $(P<0.001$; Fig. 7 c, d). 
a

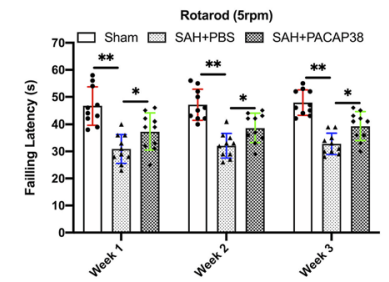

b

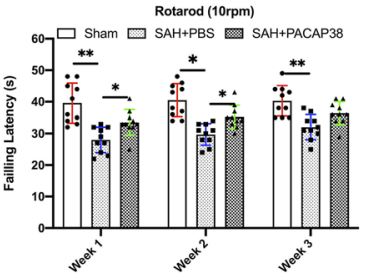

C

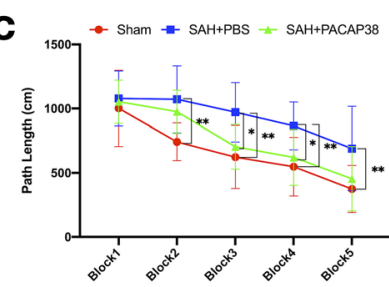

d

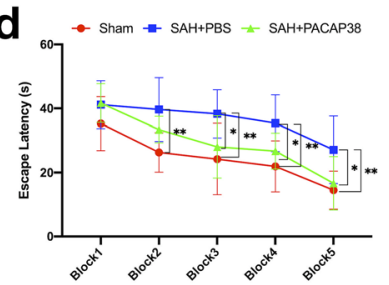

e

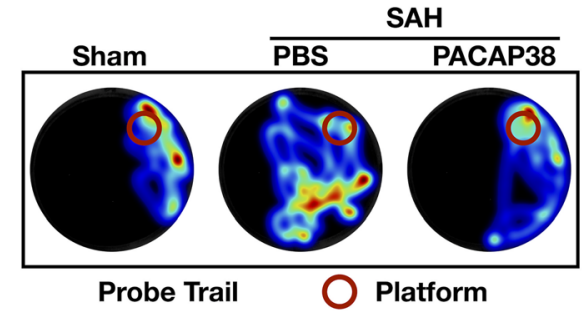

SAH

h

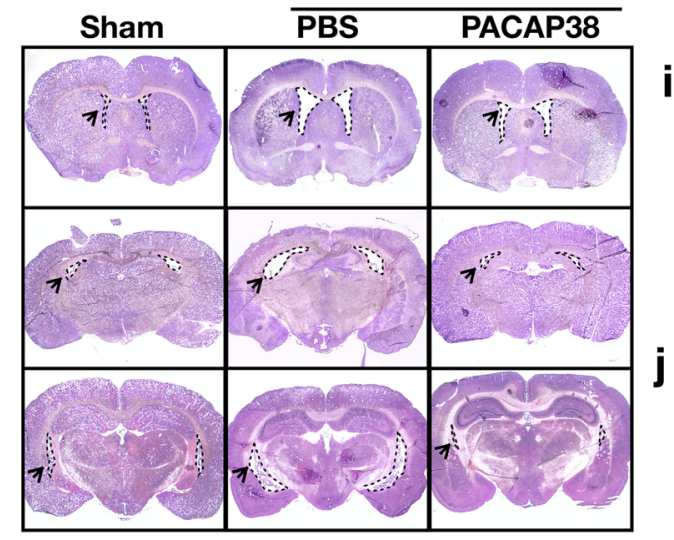

f
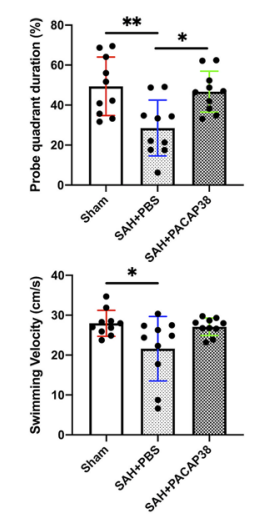

i
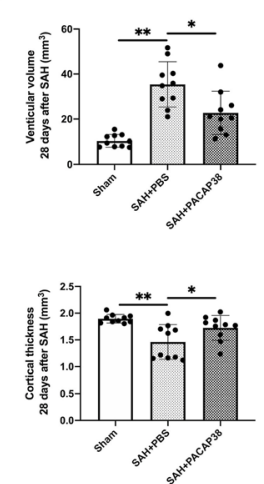

$\mathbf{k}$

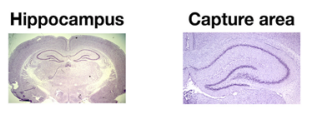

SAH

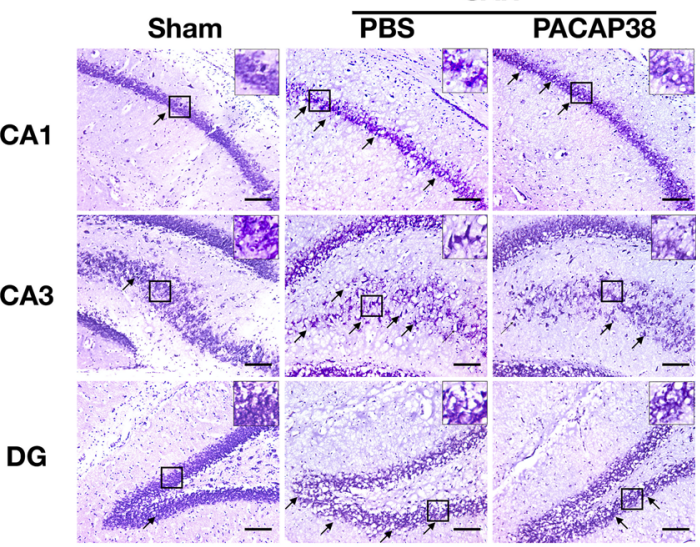

I

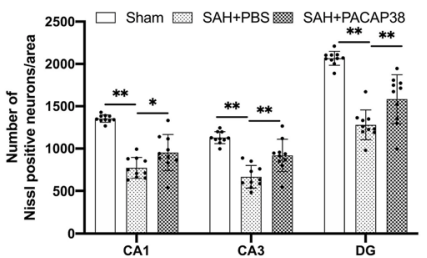

Fig. 6 Exogenous PACAP38 treatment improved long-term neurological performance and prevented hydrocephalus and neuronal degeneration at 28 days after SAH. (a, b) Rotarod test (5 RPM and 10 RPM) on the first, second, and third weeks after SAH. (c, d) Escape latency and swimming distance of Morris water maze test on days 23 to 27 after SAH. (e) Representative thermal imaging images of the probe trial. (f, g) Quantification of probe quadrant duration and swimming velocities. (hj) Representative images of coronary section at different levels, and quantification of ventricular volume $\left(\mathrm{mm}^{3}\right)$ and cortical thickness $(\mathrm{mm})$ in different groups. $(\mathrm{k}, \mathrm{l})$ Representative images and neuronal quantifications of Nissl staining in hippocampal CA1, CA3, and DG regions. Arrows indicate shrunken pyramidal or dead neurons. Scale bar $=$ $200 \mu \mathrm{m} . n=10$ /group. $* P<0.05, * * P<0.01$. One-way ANOVA, Tukey's post hoc test for (f, g, i, j). Two-way ANOVA, Tukey's post hoc test for $(a-d, i)$

0.023 compared with the PBS + control CRISPR group), as well as the PACAP38 + SUR1 activation CRISPR group $(P=$ 0.033 vs PACAP38 + control CRISPR group).

Accompanying with SUR1 activation, the expression levels of AQP4 and MMP-9 were increased but the expression levels of ZO-1 were decreased (both $P<0.016$, compared with control CRISPR groups; Fig. 8a-d). In addition, the PACAP38 treatment markedly reduced the expressions of SUR1, AQP4, and MMP-9 and preserved the expression of ZO- 1 in both the PACAP38 + control CRISPR group $(P=$ $0.024,0.004,0.019$, and 0.009 respectively compared with the 
a
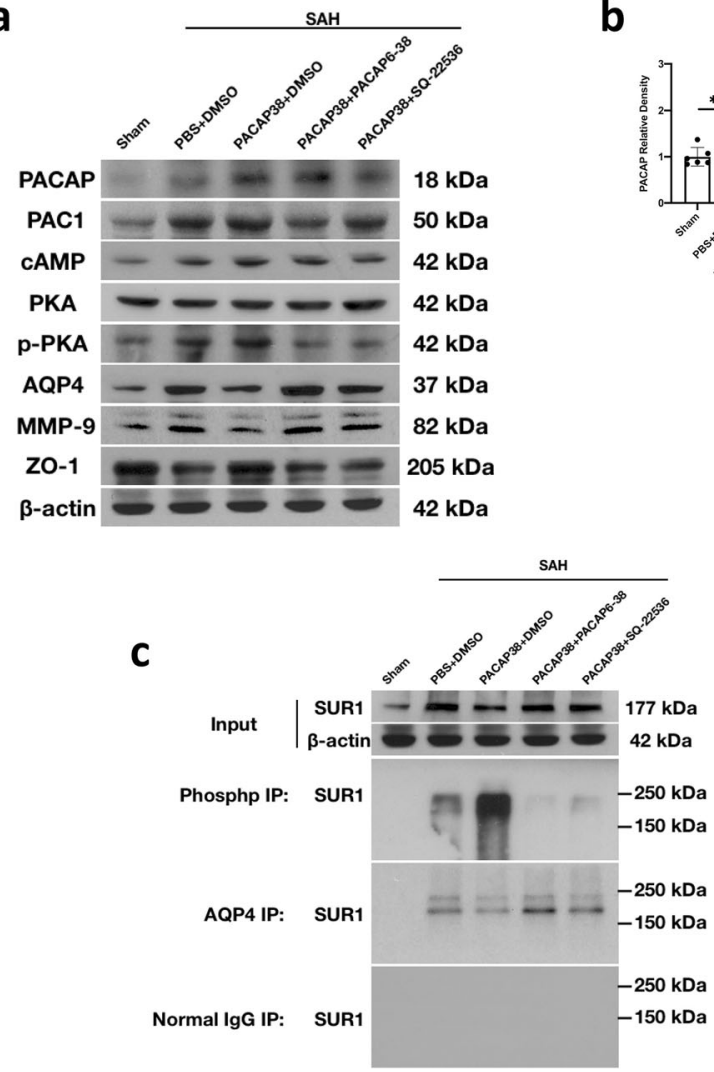

Fig. 7 PAC1/AC/cAMP/PKA pathway involved PACAP-mediated neuroprotection after SAH. (a) Representative western blot bands. (b) Densitometric quantification of PACAP, PAC1, cAMP, PKA, p-PKA, AQP4, MMP-9, and ZO-1. (c) Representative western blot bands of co-

PBS + control CRISPR group; Fig. 8a-d) and the PACAP38 + SUR1 activation CRISPR group $(P=0.017,0.009,0.025$, and 0.009 respectively compared with the PBS + SUR1 activation CRISPR group).

Co-IP showed that the activation of SUR1 in PBS and PACAP38-treated group both increased the cointegration between AQP4 and SUR1 after SAH compared with the control CRISPR groups $(P=0.037$ and 0.047, respectively) (Fig. 8f, h), whereas PACAP38 treatment in the control CRISPR and SUR1 activation CRISPR group both reduced this complex formation when compared with PBS groups $(P=0.047$ and 0.037) (Fig. 8f, h). Moreover, the phosphorylated SUR1 was significantly detectable in both PACAP38treated groups when compared with the PBS-treated groups $(P<0.001$; Fig. 8 f, g).

Taken together, the results suggested that PACAP38 may inhibit SUR1 expression by promoting the negative phosphorylation effect of p-PKA on SUR1. The increases in SUR1 degradation could decrease the expressions of AQP4 and MMP9, but preserved the ZO-1 expression after SAH (Fig. $8 i)$. b

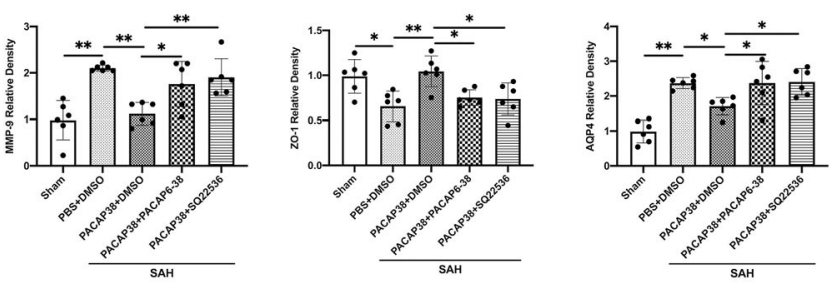

d
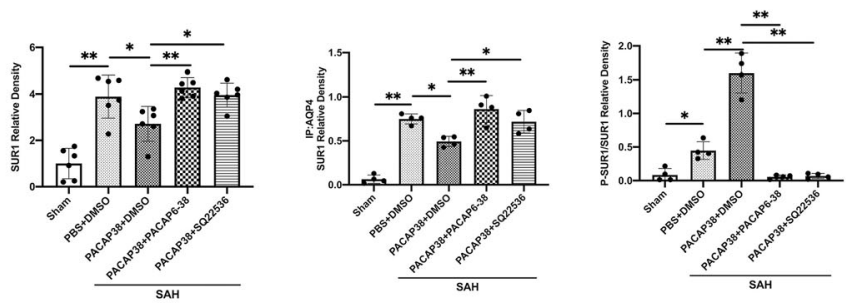

IPs. (d) Densitometric quantification of SUR1 input, SUR1 immunoprecipitated with phospho antibody, and SUR1 immunoprecipitated with AQP4. $n=4$-6/group. ${ }^{*} P<0.05$, $* * P<0.01$. One-way ANOVA, Tukey's post hoc test

\section{Discussion}

Brain edema occurs in up to $57 \%$ of patients with SAH and contributes to prolonged hospital stays and poorer outcomes $[1,4,41]$. In the present study, we are the first to demonstrate the beneficial effect of both endogenous PACAP and exogenous PACAP38 treatment in the SAH rat model. Specifically, the main findings are the following: 1) Knockout PACAP aggravated brain edema and neurological deficits in rats $24 \mathrm{~h}$ after SAH. 2) The expression of endogenous PACAP and its selective receptor, PAC1, but not receptors of VPAC1 and VPAC2, were markedly increased over time after SAH and peaked at $24 \mathrm{~h}$ post-SAH. 3) PAC1 was primarily expressed on neurons, astrocytes, and endothelial cells. Although VPAC1 was mainly expressed on neurons, VPAC2 was mainly expressed on astrocytes. 4) Exogenous PACAP38 treatment attenuated neurological deficits and brain edema $24 \mathrm{~h}$ after SAH by preserving BBB function and accelerating CSF movement/clearance. 5) The activation of the PAC1/AC/ cAMP/PKA pathway was involved in the neuroprotection of PACAP38 via phosphorylation of edema-related protein SUR1. The phosphorylation of SUR1 may promote its 

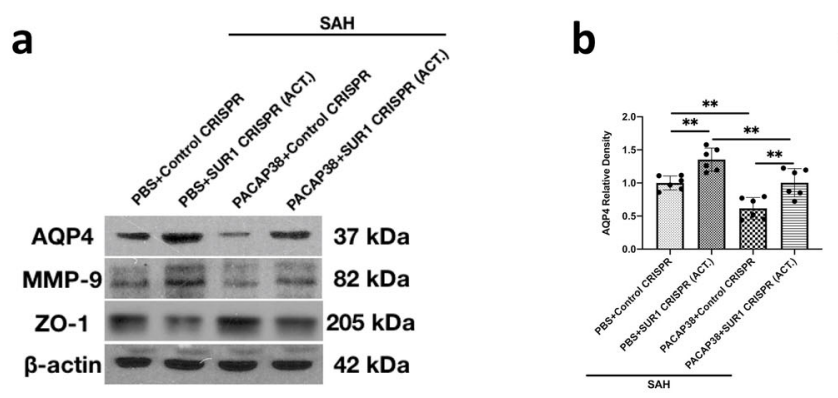

C

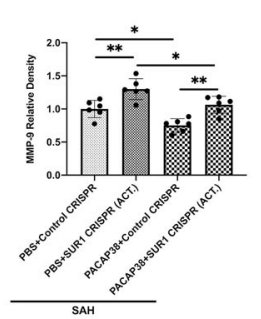

d

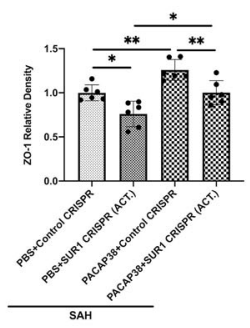

e

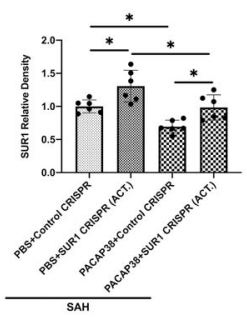

$\mathbf{f}$

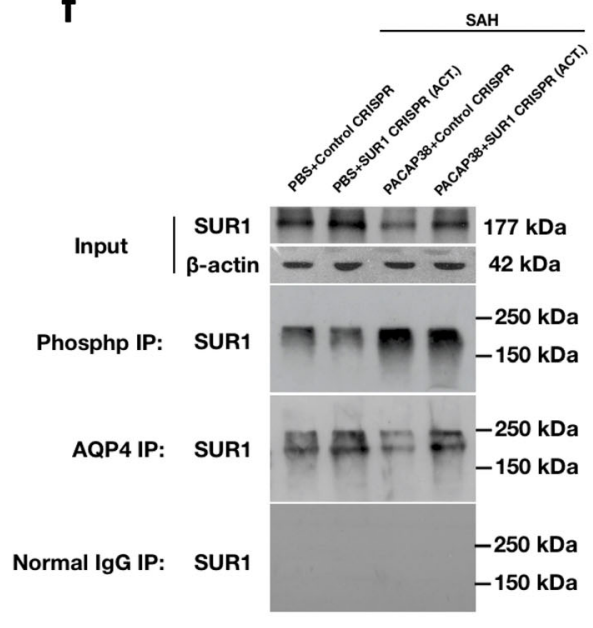

g

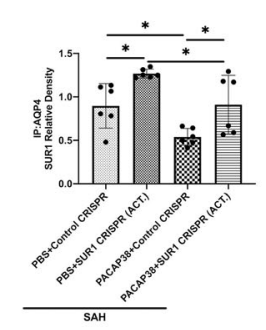

h

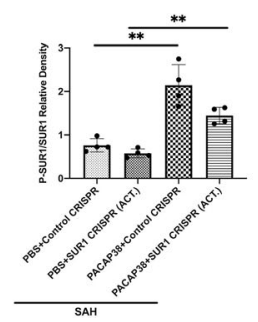

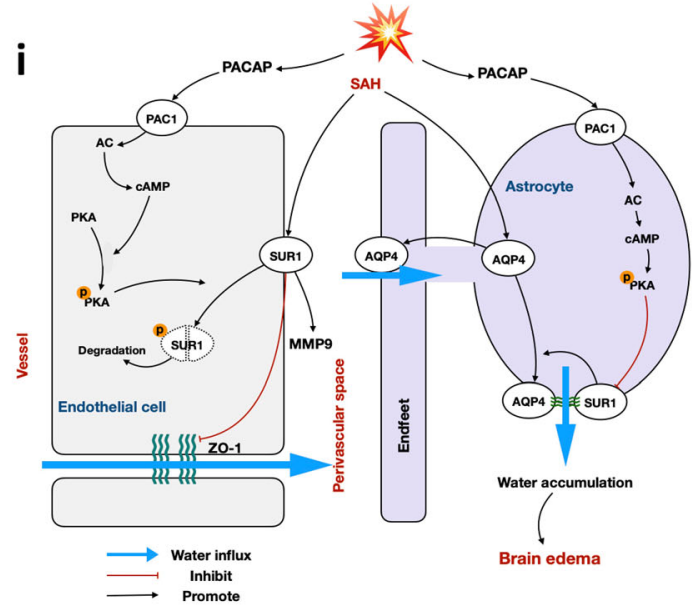

Fig. 8 PACAP attenuated brain edema formation via SUR1 regulation. (a) Representative western blot bands. (b-e) Densitometric quantification of SUR1, AQP4, MMP-9, and ZO-1. (f) Representative western blot bands of co-IPs. (g, h) Quantification of SUR1 immunoprecipitated with phospho-antibody and SUR1 immunoprecipitated with AQP4. $n=4-6 /$ group. $* P<0.05, * * P<0.01$. One-way ANOVA, Tukey's post hoc test. (i) Schema of the proposed mechanisms of PACAP-induced neuroprotection degradation. 6) The increased SUR1 expression by the SUR1 CRISPR activator facilitated AQP4 depolarization on astrocytes, leading to increased MMP-9 expression and decreased ZO-1 expression. Our findings suggest that PACAP38 may serve as a therapeutic strategy against SAH-induced brain edema by improving the BBB and glymphatic system dysfunctions.

Since first found in ovine hypothalamic tissue 4 decades ago, PACAP has been shown to play important roles in the hypothalamic-pituitary-adrenal axis and the sympathetic nervous system facing of system stress [17]. The expression of PACAP was increased following an acute brain injury, such as ischemic stroke [33, 42] and traumatic brain injury [43]. In addition to the delivery from the hypothalamus after acute brain injury, PACAP was also widely expressed on neurons, astrocytes, endothelial cells, and neural stem cells in the cortex and hippocampus [33, 42, 44, 45]. As reviewed, several harmful stimuli, such as excitotoxic glutamate, reactive oxygen species, and thrombin, could increase PACAP expression [46]. Therefore, it is not surprising that PACAP levels are upregulated in stroke. Furthermore, the PACAP has a potent neuroprotective effect in stroke [19]. PACAP is capable of improving neurological function and attenuating the increase in brain water content and brain edema volumes following both global and local ischemia in rats [21, 22]. Although higher plasma PACAP levels have been found to be associated with a better prognosis in SAH patients, the role of PACAP after SAH remains unknown [20]. In the current study, there was significantly increased endogenous PACAP expression within the ipsilateral brain hemisphere after SAH. Both endogenous and exogenous PACAP demonstrated potent antiedema effect and neuroprotection after SAH.

Among the functional receptors (PAC1, VPAC1, and VPAC2) downstream of the PACAP signaling, PAC1 was generally considered the dominant function receptor due to its high affinity with PACAP [19, 22, 26, 33]. VPAC1 and VPAC2 were expressed mainly in the peripheral nervous system, rather than the CNS, which may explain the reduced contribution of VPAC $1 / 2$ to PACAP function in the brain [18]. We found that the protein expression of PAC1 was upregulated following with the increase of PACAP after SAH, but not VPAC1/2. Such elevation of PAC1 was mainly colocalized in astrocytes and endothelial cells. The results were consistent with previous studies that found that the 
PAC1 expression on endothelial cells and astrocytes were upregulated in ischemic mice brains [33, 47]. Although the increase of PAC1 was proposed to follow the protranscription of PACAP [48], upregulation of PAC1 by exogenous PACAP38 treatment after SAH was identified in our study. In contrast, the expression of VPAC1 and VPAC2 were not significant changed after SAH. Moreover, neuroprotection provided by exogenous PACAP38 on brain edema and neurological deficits were abolished by the PAC1 inhibitor, but not the VPAC1/2 inhibitor, further confirming that PACAP functions mainly by binding to the $\mathrm{PAC} 1$ receptor after $\mathrm{SAH}$ in rats.

To be noted, both BBB dysfunction and glymphatic function impairment contribute to the early edema formation after stroke $[4,7]$. We found that the increased ICP and brain water content were associated with aggravated serum IgG and Evans blue extravasation, as well as impaired CSF movement and clearance capability (from subarachnoid/perivascular space to dcLNS and blood) in SAH rats, suggesting the involvement of $\mathrm{BBB}$ and glymphatic impairment in SAH-induced early brain edema. As previously proposed, the efflux of CSF and ISF to the dcLNs and blood may play an important role in the glymphatic system-mediated cerebral volume regulation, waste removal, and neuroimmunology [9, 49-52]. It is rational to infer that the exogenous PACAP38 treatment attenuated brain edema formation after $24 \mathrm{~h} \mathrm{SAH}$ and was associated with reduced $\mathrm{BBB}$ disruption and improved glymphatic system-mediated CSF circulation.

A previous in vitro study demonstrated that PACAP could strengthen the tight junctions between endothelial cells after glucose deprivation and oxidative stress insults [53], which is consistent with our findings of significantly higher ZO-1 expression and lower MMP-9 expression in ipsilateral brain tissues in the SAH rats treated by PACAP38. The preservation of $\mathrm{BBB}$ function resulted in reduced tissue IgG extravasation and EB concentration. In addition, ion/water channels play important roles in the pathogenesis of BBB and glymphatic system dysfunction after stroke [4, 10]. Stroke-induced ATP deficiency immediately upregulated SUR1 on endothelial cells [13] and astrocytes [54], leading to edema formation. Somatic SUR1 was found to mediate astrocytic swelling by combining with AQP4 [11]. AQP4, an aquaporin protein located on astrocytic end feet, maintains high polarization near the vasculature to facilitate the water flux exchange between the perivascular space and the parenchyma [51]. The formation of the SUR1/AQP4 complex on astrocytic soma may result in the depolarization of AQP4 and impairment of glymphatic function [11]. Previous studies revealed the depolarized AQP4 was generally found after acute brain injury, such as cerebral ischemia [55], traumatic brain injury [56], and cerebral hemorrhage [9]. It was also proposed that the AQP4 depolarization-mediated glymphatic dysfunction could last from days to a month after cerebral hemorrhage [9, 57].
When we used SUR1 activation CRISPR to increase SUR1 expression in the brain after SAH, the upregulation of SUR1 further exacerbated brain MMP-9 elevation and ZO-1 reduction, as well as promoted the combination of brain SUR1/ AQP4 in SAH rats.

PACAP was found on both neural and nonneural cells [25, 26] as a potent modulator of ion/water channels, including potassium channels, sodium channels, calcium-dependent potassium channels, and aquaporins. Our study showed that PACAP38 treatment reduced the elevation of brain protein levels of MMP-9, SUR1, and AQP4, but preserved the ZO-1 expression after SAH, which may explain the neuroprotection exerted on short-term outcome, including neurobehavioral function, BBB, and cerebrospinal fluid circulation disorders, as well as long-term hydrocephalus and neuronal loss. However, these effects were significant reversed by SUR1 activation CRISPR. Taken together, our findings suggested that PACAP38 attenuated BBB disruption and AQP4 depolarization-mediated glymphatic dysfunction by SUR1 downregulation.

We further investigated the signaling pathway underlying PACAP-mediated SUR1 downregulation after SAH. The classical AC/cAMP/PKA axis was suggested to be one of the key signaling pathways involved in the PACAPmediated regulator of ion/water channels $[25,26]$. We demonstrated that the activation of the AC/cAMP/PKA axis underlined PACAP-provided neuroprotection by promoting SUR1 phosphorylation. In general, the PACAP-induced modulation effect on channel proteins varied from different cell localizations and physiological functions [25]. Also, the PKAinduced phosphorylation effect on SUR1 channels has distinct roles on its activity [27, 58]. However, it was reported that marked alteration of the PKA-mediated phosphorylation may lead to the inactivation of SUR1 channel in pancreatic cell [58]. In our study, PACAP-mediated negative regulation of SUR1 was most likely through the activation of PAC1/AC/ cAMP/PKA signaling, which promoted the SUR1 degradation by its phosphorylation. The administration of PAC1 inhibitor or AC inhibitor abolished the effects of PACAP38 treatment on protein levels of $\mathrm{PAC} 1 / \mathrm{AC} / \mathrm{cAMP} / \mathrm{PKA}$ signaling and SUR1 expressions in brain tissues after SAH.

\section{Limitations}

Several limitations remain in this study. First, PACAP was also shown to have potent anti-neuronal apoptotic effects after ischemic stroke [16]. We could not exclude the contribution of anti-apoptotic mechanisms to both short- and long-term neurological behavior improvements in the current study. Second, the methods of glymphatic function assessment were limited. We only used cisterna magna injection of EB to indirectly estimate the CSF movement and clearance through the 
glymphatic system. Despite the AQP4 perivascular proportion being measured, which could also partly help elucidate the glymphatic system function, further studies are still encouraged to validate the effects of PACAP on glymphatic pathway function using CSF tracer and/or MRI technique. Third, although control CRISPR was used to exclude impact carried by the potential off-target effects of PACAP and SUR1 CRISPR to the results, there may still exist some unpredictable offtargets effect that cannot be revealed by the neurological test or western blot. Future studies are needed to further validate the mechanism use a transgenic animal model. Lastly, the PACAP is a vasodilator, which may potentially change cerebral blood flow and further exacerbate brain edema. However, such vasodilatory effects seem to be dose-dependent. A previous study showed that only intravenous dosages higher than $50 \mathrm{nmol} / \mathrm{kg}$ would cause a long-lasting decline of cerebral blood flow [59]. Nevertheless, a much lower dose $(6.6 \mathrm{nmol} / \mathrm{kg}$, i.p. $)$ was applied in the present study, and we found that PACAP treatment significantly reduced brain edema and improved neurological outcomes in rats after SAH.

\section{Conclusion}

Exogenous PACAP38 treatment markedly reduced brain edema formation and improved neurological deficits by preserving $\mathrm{BBB}$ and glymphatic function after $\mathrm{SAH}$ in rats. The PAC1/ AC/cAMP/PKA signaling pathway was involved in the PACAP-provided neuroprotection by downregulating SUR1 in brain. These findings suggested that PACAP is a potent neuroprotective peptide that may serve as a potential clinical treatment to relieve brain edema in patients with SAH.

Acknowledgments: This study was supported by grants from the National Institutes of Health (NS081740 and NS082184) to John H. Zhang and the Key Program of Science and Technology Development of Zhejiang Province (2017C03021) and National Natural Science Foundation of China (81870916 and 81901244) to Jianmin Zhang and Keren Zhou.

Required Author Forms Disclosure forms provided by the authors are available with the online version of this article.

\section{Compliance with Ethical Standards}

Conflict of Interest The authors declare that they have no conflict of interest.

\section{References}

1. Rass V, Ianosi BA, Wegmann A, Gaasch M, Schiefecker AJ, Kofler M, et al. Delayed resolution of cerebral edema is associated with poor outcome after nontraumatic subarachnoid hemorrhage. Stroke. 2019;50(4):828-36.
2. Ahn SH, Savarraj JP, Pervez M, Jones W, Park J, Jeon SB, et al. The subarachnoid hemorrhage early brain edema score predicts delayed cerebral ischemia and clinical outcomes. Neurosurgery. 2017.

3. Zhang C, Jiang M, Wang WQ, Zhao SJ, Yin YX, Mi QJ, et al. Selective mGluR1 negative allosteric modulator reduces bloodbrain barrier permeability and cerebral edema after experimental subarachnoid hemorrhage. Transl Stroke Res. 2019.

4. Stokum JA, Gerzanich V, Simard JM. Molecular pathophysiology of cerebral edema. J Cereb Blood Flow Metab. 2016;36(3):513-38.

5. Hayman EG, Wessell A, Gerzanich V, Sheth KN, Simard JM. Mechanisms of global cerebral edema formation in aneurysmal subarachnoid hemorrhage. Neurocrit Care. 2017;26(2):301-10.

6. Norenberg MD. Astrocyte responses to CNS injury. J Neuropathol Exp Neurol. 1994;53(3):213-20.

7. Mestre H, Du T, Sweeney AM, Liu G, Samson AJ, Peng W, et al. Cerebrospinal fluid influx drives acute ischemic tissue swelling. Science. 2020;367(6483).

8. Gaberel T, Gakuba C, Goulay R, Martinez De Lizarrondo S, Hanouz JL, Emery E, et al. Impaired glymphatic perfusion after strokes revealed by contrast-enhanced MRI: a new target for fibrinolysis? Stroke. 2014;45(10):3092-6.

9. Ding Y, Zhang T, Wu G, McBride DW, Xu N, Klebe DW, et al. Astrogliosis inhibition attenuates hydrocephalus by increasing cerebrospinal fluid reabsorption through the glymphatic system after germinal matrix hemorrhage. Exp Neurol. 2019;320:113003.

10. Mader S, Brimberg L. Aquaporin-4 water channel in the brain and its implication for health and disease. Cells. 2019;8(2).

11. Stokum JA, Kwon MS, Woo SK, Tsymbalyuk O, Vennekens R, Gerzanich V, et al. SUR1-TRPM4 and AQP4 form a heteromultimeric complex that amplifies ion/water osmotic coupling and drives astrocyte swelling. Glia. 2018;66(1):108-25.

12. Mori K, Miyazaki M, Iwase H, Maeda M. Temporal profile of changes in brain tissue extracellular space and extracellular ion $(\mathrm{Na}(+), \mathrm{K}(+))$ concentrations after cerebral ischemia and the effects of mild cerebral hypothermia. J Neurotrauma. 2002;19(10):126170.

13. Khanna A, Kahle KT, Walcott BP, Gerzanich V, Simard JM. Disruption of ion homeostasis in the neurogliovascular unit underlies the pathogenesis of ischemic cerebral edema. Transl Stroke Res. 2014;5(1):3-16.

14. Rajasekaran SA, Beyenbach KW, Rajasekaran AK. Interactions of tight junctions with membrane channels and transporters. Biochim Biophys Acta. 2008;1778(3):757-69.

15. Simard JM, Geng Z, Woo SK, Ivanova S, Tosun C, Melnichenko L, et al. Glibenclamide reduces inflammation, vasogenic edema, and caspase-3 activation after subarachnoid hemorrhage. J Cereb Blood Flow Metab. 2009;29(2):317-30.

16. Reglodi D, Vaczy A, Rubio-Beltran E, MaassenVanDenBrink A. Protective effects of PACAP in ischemia. J Headache Pain. 2018;19(1):19.

17. Rudecki AP, Gray SL. PACAP in the defense of energy homeostasis. Trends Endocrinol Metab. 2016;27(9):620-32.

18. Vaudry D, Falluel-Morel A, Bourgault S, Basille M, Burel D, Wurtz $\mathrm{O}$, et al. Pituitary adenylate cyclase-activating polypeptide and its receptors: 20 years after the discovery. Pharmacol Rev. 2009;61(3):283-357.

19. Chen Y, Samal B, Hamelink CR, Xiang CC, Chen Y, Chen M, et al. Neuroprotection by endogenous and exogenous PACAP following stroke. Regul Pept. 2006;137(1-2):4-19.

20. Jiang L, Wang WH, Dong XQ, Yu WH, Du Q, Yang DB, et al. The change of plasma pituitary adenylate cyclase-activating polypeptide levels after aneurysmal subarachnoid hemorrhage. Acta Neurol Scand. 2016;134(2):131-9.

21. Dong Y, He C, Han X, Wang CH, Lu CL. Experimental study of pituitary adenylate cyclase activating polypeptide on relieving brain 
edema induced by ischemia in rats. Zhongguo Ying Yong Sheng Li Xue Za Zhi. 2002;18(2):121-3.

22. Nakamachi T, Ohtaki H, Yofu S, Dohi K, Watanabe J, Mori H, et al. Endogenous pituitary adenylate cyclase activating polypeptide is involved in suppression of edema in the ischemic brain. Acta Neurochir Suppl. 2010;106:43-6.

23. Banki E, Hajna Z, Kemeny A, Botz B, Nagy P, Bolcskei K, et al. The selective $\mathrm{PAC} 1$ receptor agonist maxadilan inhibits neurogenic vasodilation and edema formation in the mouse skin. Neuropharmacology. 2014;85:538-47.

24. Scuderi S, D'Amico AG, Castorina A, Imbesi R, Carnazza ML, D'Agata V. Ameliorative effect of PACAP and VIP against increased permeability in a model of outer blood retinal barrier dysfunction. Peptides. 2013;39:119-24.

25. Johnson GC, May V, Parsons RL, Hammack SE. Parallel signaling pathways of pituitary adenylate cyclase activating polypeptide (PACAP) regulate several intrinsic ion channels. Ann N Y Acad Sci. 2019;1455(1):105-12.

26. Nakamachi T, Ohtaki H, Seki T, Yofu S, Kagami N, Hashimoto H, et al. PACAP suppresses dry eye signs by stimulating tear secretion. Nat Commun. 2016;7:12034

27. Beguin P, Nagashima K, Nishimura M, Gonoi T, Seino S. PKAmediated phosphorylation of the human K(ATP) channel: separate roles of Kir6.2 and SUR1 subunit phosphorylation. EMBO J. 1999;18(17):4722-32.

28. Kang G, Chepurny OG, Malester B, Rindler MJ, Rehmann H, Bos JL, et al. cAMP sensor Epac as a determinant of ATP-sensitive potassium channel activity in human pancreatic beta cells and rat INS-1 cells. J Physiol. 2006;573(Pt 3):595-609.

29. Chu H, Huang C, Ding H, Dong J, Gao Z, Yang X, et al. Aquaporin-4 and cerebrovascular diseases. Int J Mol Sci. 2016;17(8).

30. Zhang T, Wu P, Budbazar E, Zhu Q, Sun C, Mo J, et al. Mitophagy reduces oxidative stress via Keap1 (Kelch-like epichlorohydrinassociated protein 1)/Nrf2 (nuclear factor-E2-related factor 2)/ PHB2 (prohibitin 2) pathway after subarachnoid hemorrhage in rats. Stroke. 2019;50(4):978-88.

31. Zuo G, Zhang T, Huang L, Araujo C, Peng J, Travis Z, et al. Activation of TGR5 with INT-777 attenuates oxidative stress and neuronal apoptosis via cAMP/PKCepsilon/ALDH2 pathway after subarachnoid hemorrhage in rats. Free Radic Biol Med. 2019;143: 441-53.

32. Perez-Pinera P, Kocak DD, Vockley CM, Adler AF, Kabadi AM, Polstein LR, et al. RNA-guided gene activation by CRISPR-Cas9based transcription factors. Nat Methods. 2013;10(10):973-6.

33. Lin $\mathrm{CH}$, Chiu L, Lee HT, Chiang CW, Liu SP, Hsu YH, et al. PACAP38/PAC1 signaling induces bone marrow-derived cells homing to ischemic brain. Stem Cells. 2015;33(4):1153-72.

34. Pang J, Peng J, Matei N, Yang P, Kuai L, Wu Y, et al. Apolipoprotein $\mathrm{E}$ exerts a whole-brain protective property by promoting M1? Microglia quiescence after experimental subarachnoid hemorrhage in mice. Transl Stroke Res. 2018;9(6):654-68.

35. Xie Y, Li YJ, Lei B, Kernagis D, Liu WW, Bennett ER, et al. Sex differences in gene and protein expression after intracerebral hemorrhage in mice. Transl Stroke Res. 2019;10(2):231-9.

36. Wolf MS, Chen Y, Simon DW, Alexander H, Ross M, Gibson GA, et al. Quantitative and qualitative assessment of glymphatic flux using Evans blue albumin. J Neurosci Methods. 2019;311:436-41.

37. Siler DA, Gonzalez JA, Wang RK, Cetas JS, Alkayed NJ. Intracisternal administration of tissue plasminogen activator improves cerebrospinal fluid flow and cortical perfusion after subarachnoid hemorrhage in mice. Transl Stroke Res. 2014;5(2):22737.

38. Luh C, Feiler S, Frauenknecht K, Meyer S, Lubomirov LT, Neulen A, et al. The contractile apparatus is essential for the integrity of the blood-brain barrier after experimental subarachnoid hemorrhage. Transl Stroke Res. 2019;10(5):534-45.

39. Zhang Y, Ding Y, Lu T, Zhang Y, Xu N, Yu L, et al. Bliverdin reductase-A improves neurological function in a germinal matrix hemorrhage rat model. Neurobiol Dis. 2018;110:122-32.

40. Okada T, Enkhjargal B, Travis ZD, Ocak U, Tang J, Suzuki H, et al. FGF-2 attenuates neuronal apoptosis via FGFR3/PI3k/Akt signaling pathway after subarachnoid hemorrhage. Mol Neurobiol. 2019;56(12):8203-19.

41. Fang YJ, Mei SH, Lu JN, Chen YK, Chai ZH, Dong X, et al. New risk score of the early period after spontaneous subarachnoid hemorrhage: for the prediction of delayed cerebral ischemia. CNS Neurosci Ther. 2019;25(10):1173-81.

42. Matsumoto M, Nakamachi T, Watanabe J, Sugiyama K, Ohtaki H, Murai N, et al. Pituitary adenylate cyclase-activating polypeptide (PACAP) is involved in adult mouse hippocampal neurogenesis after stroke. J Mol Neurosci. 2016;59(2):270-9.

43. Toth D, Tamas A, Reglodi D. The neuroprotective and biomarker potential of PACAP in human traumatic brain injury. Int J Mol Sci. 2020;21(3).

44. Waschek JA. Multiple actions of pituitary adenylyl cyclase activating peptide in nervous system development and regeneration. Dev Neurosci. 2002;24(1):14-23.

45. Waschek JA. VIP and PACAP: neuropeptide modulators of CNS inflammation, injury, and repair. Br J Pharmacol. 2013;169(3):51223.

46. Reglodi D, Tamas A, Jungling A, Vaczy A, Rivnyak A, Fulop BD, et al. Protective effects of pituitary adenylate cyclase activating polypeptide against neurotoxic agents. Neurotoxicology. 2018;66: 185-94.

47. Nakamachi T, Farkas J, Kagami N, Wada Y, Hori M, Tsuchikawa $\mathrm{D}$, et al. Expression and distribution of pituitary adenylate cyclaseactivating polypeptide receptor in reactive astrocytes induced by global brain ischemia in mice. Acta Neurochir Suppl. 2013;118: 55-9.

48. Riek-Burchardt M, Kolodziej A, Henrich-Noack P, Reymann KG, Hollt V, Stumm R. Differential regulation of CXCL12 and PACAP mRNA expression after focal and global ischemia. Neuropharmacology. 2010;58(1):199-207.

49. Jessen NA, Munk AS, Lundgaard I, Nedergaard M. The glymphatic system: a beginner's guide. Neurochem Res. 2015;40(12):2583-99.

50. Plog BA, Nedergaard M. The glymphatic system in central nervous system health and disease: past, present, and future. Annu Rev Pathol. 2018;13:379-94.

51. Rasmussen MK, Mestre H, Nedergaard M. The glymphatic pathway in neurological disorders. Lancet Neurol. 2018;17(11):101624.

52. Maloveska M, Danko J, Petrovova E, Kresakova L, Vdoviakova K, Michalicova A, et al. Dynamics of Evans blue clearance from cerebrospinal fluid into meningeal lymphatic vessels and deep cervical lymph nodes. Neurol Res. 2018;40(5):372-80.

53. Wilhelm I, Fazakas C, Tamas A, Toth G, Reglodi D, Krizbai IA. PACAP enhances barrier properties of cerebral microvessels. J Mol Neurosci. 2014;54(3):469-76.

54. Pasantes-Morales H, Vazquez-Juarez E. Transporters and channels in cytotoxic astrocyte swelling. Neurochem Res. 2012;37(11): 2379-87.

55. Wang M, Iliff JJ, Liao Y, Chen MJ, Shinseki MS, Venkataraman A, et al. Cognitive deficits and delayed neuronal loss in a mouse model of multiple microinfarcts. J Neurosci. 2012;32(50):17948-60.

56. Iliff JJ, Nedergaard M. Is there a cerebral lymphatic system? Stroke. 2013;44(6 Suppl 1):S93-5.

57. Luo C, Yao X, Li J, He B, Liu Q, Ren H, et al. Paravascular pathways contribute to vasculitis and neuroinflammation after subarachnoid hemorrhage independently of glymphatic control. Cell Death Dis. 2016;7:e2160. 
58. Light PE, Manning Fox JE, Riedel MJ, Wheeler MB. Glucagonlike peptide-1 inhibits pancreatic ATP-sensitive potassium channels via a protein kinase A- and ADP-dependent mechanism. Mol Endocrinol. 2002;16(9):2135-44.

59. Ohtaki H, Dohi K, Yofu S, Nakamachi T, Kudo Y, Endo S, et al. Effect of pituitary adenylate cyclase-activating polypeptide 38
(PACAP38) on tissue oxygen content-treatment in central nervous system of mice. Regul Pept. 2004;123(1-3):61-7.

Publisher's Note Springer Nature remains neutral with regard to jurisdictional claims in published maps and institutional affiliations. 